\title{
On optimal trajectory for the multi-period evolution of FTTx networks
}

\author{
Mariusz Mycek $^{1}$ (D) $\cdot$ Mateusz Żotkiewicz ${ }^{1}$
}

Published online: 25 August 2018

(c) The Author(s) 2018

\begin{abstract}
We propose a business/cost model for long-period changes of customer demands in FTTx networks. We show how to exploit this model in the formulation of a MIP optimization problem that, for a given sequence of customer demand vectors provides (when solved to the optimality) the absolute benchmark. The model is relatively complicated and cannot be directly applied to the optimization of real-world networks without a number of algorithmic tricks that are discussed in this paper. The presented approach has been implemented and tested against an example network instance. Test results are given and discussed in the paper.
\end{abstract}

Keywords FTTx $\cdot$ FTTH $\cdot$ Optical access network $\cdot$ Multi-period optimization

\section{Introduction}

Deployment of the FTTx optical access network (FTTxOAN) is a large business challenge that incurs considerable capital and operational expenditures and thus it requires careful planning using advanced optimization methods and tools. The FTTx network design problem has been intensively studied over the last two decades. Unfortunately majority of published models regarding the considered issue lack some important features which makes them impractical from the industrial viewpoint, as they were usually proposed before massive industrial experience in rolling-out was available. In effect, a lot of assumptions adopted in those papers lead to oversimplifications. For instance in [6], only one split with fixed ratio is allowed. In [26,31], trenching is considered separately for each cable; thus, parallel cables cannot be buried in one trench. Another example is [10], in which the split ratio is fixed. On the other hand, some models are very complex. For example in [22], even the cost and attenuation of splices are considered. Unfortunately, such models can be utilized for only relatively small use cases. In $[9,17,18]$, neat MIP models are presented and practically justified. Another recent work is [1] covering the last access part of FTTH net-

Mariusz Mycek

mariusz@tele.pw.edu.pl

Mateusz Żotkiewicz

mzotkiew@tele.pw.edu.pl

1 Institute of Telecommunications, Warsaw University of Technology, Nowowiejska 15/19, 00-665 Warszawa, Poland work; thus, not considering splitting and OLT costs. Still, the detailed view of the fiber splicing problem presented in [1] is definitely worth noting. Finally, a comprehensive summary of MIP approaches to FTTH network design can be found in [16].

Recently a number of heuristics have been introduced in the field. An approach presented in [3] is based on clustering, uses Tabu search, and has been enhanced with resiliency in [2]. On the other hand, approach presented in [35] is based on beam search [5], has been enhanced with uncertainty issues in [32], and upgraded with the MIP polishing in [23]. This last approach of mixing the MIP methodology with heuristics proved to be very efficient and has been recently used also in [12].

A question how to optimally accommodate long-period changes of customer demands in FTTx networks has also attracted attention of researchers. Due to the long network lifetime, the planing is a multi-step process-after the initial set-up-it is performed regularly on the period-by-period basis. In every step, the future network evolution is decided, taking into account the current state and the near-future requirements, anticipated on the basis of historical data and the assumed model of the customer arrival process. In [26], the future growth of demand volumes was addressed. However, in their research, the whole deployment process was not divided into phases. Similar assumption was adapted in [17] in which a polyhedral model was used to express demand volumes. On the other hand, in [33,34] multiperiod approach to the deployment of FTTH networks was used. However, 
is was assumed that a network design is given and only its exact execution is optimized and divided into phases.

Unfortunately, the quality of results the reported methods provide is difficult to evaluate and to compare. The fundamental obstacles are the lack of a common cost model and an optimal benchmark solution they could refer to. The originality of our paper is that it identifies and aims at filling that gap. It proposes business and cost models suitable for analysis of multi-period network evolution and formulates the MIP optimization problem to designate the most cost-effective evolution pattern for a given sequence of demand vectors. The models presented are general and can be exploited (still are not restricted to) to formulation of both optimization and decision problems. The presented optimization problem cannot be directly exploited to plan future development of FTTx network still, as it provides the benchmark solution, it can assist such planning in the following ways. First, it can help in a posteriori evaluation of design decisions made by network operators during the analyzed period of time. Second, it can be exploited in a priori analysis how the considered deployment rules would behave w.r.t. different customer arrival trajectories. Third, it can help in identification of decisions and structural/procedural assumptions that are of crucial value (or can be simply neglected) in the decision problem.

The approach presented in the paper has been implemented and tested against a single example of real network instance. Results of selected numerical experiments, which illustrate how particular design decisions can influence the optimal solution, are given and analyzed in the paper.

The paper is organized as follows. Section 2 shows an introductory example. Section 3 introduces a model of FTTx networks that provides necessary foundations for formulation of the optimization problem. Section 4 considers business environment of the FTTx network deployment, shows a suitable business model then introduces and justifies the cost model used in the optimization. Section 5 shows the optimization problem formulation itself; goals and results of selected numerical experiments are given and analyzed in Sect. 6. Finally, Sect. 7 summarizes and concludes the paper.

\section{Example case}

To recognize a problem considered in this paper, we start with the example network presented in Fig. 1, originally introduced in [34]. The network provides the FTTH access for two Multi-Dwelling Units (MDU), of 90 flats each-MDU $U_{A}$ (at the top of the figure) and $M D U_{B}$ (at the bottom). We assume that the utilized FTTx technology allows for the maximum split of 1:64, and that the network has been designed in a way that all potential clients can connect. By the design, the Access Point (AP) dedicated to each MDU can host one split-

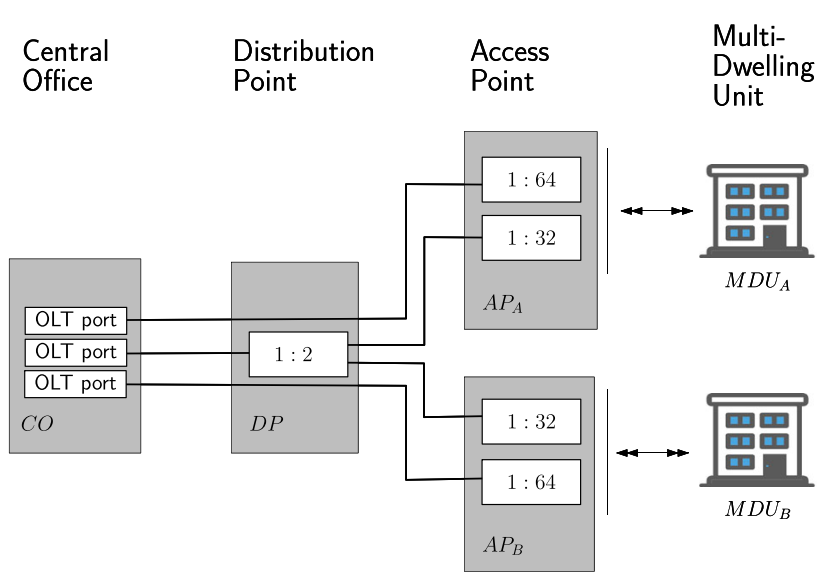

Fig. 1 Example FTTH network

ter with 64 outputs (denoted by 1:64) and one splitter with 32 outputs (1:32).

Now we inspect a few example deployments corresponding to various demand patterns. Generally, the deployment follows the design still, to generate savings, only the necessary equipment is installed. Thus, when the take-up rate is equal to 1 (we refer to this situation as pattern $P_{I}$ ), all the splitters, which have been located in the APs by the design, should be deployed; additionally one two-output splitter should be installed in the Distribution Point (DP) to connect the 32-output splitters at the APs to a single OLT port; obviously, three OLT ports are utilized, in total, at the Central Office $(C O)$. In the case when the take-up rate is $\frac{2}{3}$ (pattern $P_{I I}$ ) only the 64-output splitters are needed and two OLT ports should be utilized. Finally, with the take-up rate equal to $\frac{1}{3}$ (pattern $P_{I I I}$ ) only the 32-output splitters (together with a two-output splitter in $D P$ ) and one OLT port should be used.

We need to introduce some formalism to organize and generalize further analysis. Observe that, under mentioned assumptions, vector $x=\left(x_{A}^{64}, x_{A}^{32}, x_{B}^{64}, x_{B}^{32}, x_{C}^{2}\right)$ of binary elements expressing presence/absence of 1:64 and 1:32 splitters in $A P_{A}$ and $A P_{B}$ access points and of $1: 2$ splitter in $D P$ distribution point, unambiguously distinguishes a complete network configuration. As an example, vector $x=$ $(1,0,0,1,1)$ denotes a network configuration with one 1:64 splitter in $A P_{A}$ and one 1:32 splitter in $A P_{B}$ (with its supporting 1:2 splitter in $D P$ ). The set of all admissible network configurations is denoted by $X$ (notice that $|X|=16$, because $x_{C}^{2}$ is fully determined by values of $x_{A}^{32}$ and $x_{B}^{32}$ configuration components); each configuration $x \in X$ is characterized by the service capacity vector $s(x)=\left(s_{A}(x), s_{B}(x)\right)$ representing the number of active ports provided in, respectively, $A P_{A}$ 
Table 1 Configurations of the example network

\begin{tabular}{|c|c|c|c|c|c|c|c|c|c|}
\hline & \multicolumn{5}{|l|}{$x$} & \multicolumn{2}{|l|}{$\mathrm{s}(\mathrm{x})$} & \multicolumn{2}{|c|}{$n(x)$} \\
\hline & $\overline{x_{A}^{64}}$ & $x_{A}^{32}$ & $x_{B}^{64}$ & $x_{B}^{32}$ & $x_{C}^{2}$ & $\overline{s_{A}}$ & $\overline{s_{B}}$ & $\overline{n^{H}}$ & $n^{D}$ \\
\hline$x_{0}$ & 0 & 0 & 0 & 0 & 0 & 0 & 0 & 0 & 0 \\
\hline$x_{1}$ & 0 & 0 & 0 & 1 & 1 & 0 & 32 & 1 & 1 \\
\hline$x_{2}$ & 0 & 0 & 1 & 0 & 0 & 0 & 64 & 1 & 1 \\
\hline$x_{3}$ & 0 & 0 & 1 & 1 & 1 & 0 & 96 & 2 & 2 \\
\hline$x_{4}$ & 0 & 1 & 0 & 0 & 1 & 32 & 0 & 1 & 1 \\
\hline$x_{5}$ & 0 & 1 & 0 & 1 & 1 & 32 & 32 & 1 & 2 \\
\hline$x_{6}$ & 0 & 1 & 1 & 0 & 1 & 32 & 64 & 2 & 2 \\
\hline$x_{7}$ & 0 & 1 & 1 & 1 & 1 & 32 & 96 & 2 & 3 \\
\hline$x_{8}$ & 1 & 0 & 0 & 0 & 0 & 64 & 0 & 1 & 1 \\
\hline$x_{9}$ & 1 & 0 & 0 & 1 & 1 & 64 & 32 & 2 & 2 \\
\hline$x_{10}$ & 1 & 0 & 1 & 0 & 0 & 64 & 64 & 2 & 2 \\
\hline$x_{11}$ & 1 & 0 & 1 & 1 & 1 & 64 & 96 & 3 & 3 \\
\hline$x_{12}$ & 1 & 1 & 0 & 0 & 1 & 96 & 0 & 2 & 2 \\
\hline$x_{13}$ & 1 & 1 & 0 & 1 & 1 & 96 & 32 & 2 & 3 \\
\hline$x_{14}$ & 1 & 1 & 1 & 0 & 1 & 96 & 64 & 3 & 3 \\
\hline$x_{15}$ & 1 & 1 & 1 & 1 & 1 & 96 & 96 & 3 & 4 \\
\hline
\end{tabular}

and $A P_{B}$ access points ${ }^{1}$ — and by the cost of its deployment $c(x) \in \mathbb{R}_{+}$.

Let $d=\left(d_{A}, d_{B}\right)$ denote a demand vector, i.e., the number of optical signals that the network must provide in, respectively, $A P_{A}$ and $A P_{B}$ access points-thus demand patterns $P_{I}, P_{I I}$, and $P_{I I I}$ can be represented by vectors $d_{I}=(90,90), d_{I I}=(60,60)$, and $d_{I I I}=(30,30)$; set of all considered demand vectors is designated by $\mathfrak{D}$. For each subset of demand vectors $D \subseteq \mathfrak{D}$, we identify a set of compatible network configurations $X(D) \subseteq X$, which are able to satisfy every demand vector of the subset, i.e., such that $s(x) \succeq d, x \in X(d), d \in D$. If set $D$ is a singleton, i.e., $D=\{d\}$, we denote a set of its compatible configurations by $X(d)$.

Taking advantage of the introduced notions, in Table 1, we characterize all admissible configurations of the example network. Each configuration $x \in X$, is described in-there in terms of a vector of installed splitters $x$, provided service capacity $s(x)$, and finally, the number of optical fibres exploited in $C O-D P$ and $D P-A P$ relations-in order, $n^{H}(x)$ and $n^{D}(x)$.

Observe, that service capacities of considered network configurations split $A P_{A}$ and $A P_{B}$ components of demand vectors into sequences of disjoint ranges; these are, respectively, $R_{A}=\left\{R_{A}^{0}=0, R_{A}^{1}=1 \ldots 32, R_{A}^{2}=33 \ldots 64, R_{A}^{3}=\right.$ $65 \ldots 96\}$ and $R_{B}=\left\{R_{B}^{0}=0, R_{B}^{1}=1 \ldots 32, R_{B}^{2}=\right.$ $33 \ldots 64, R_{B}^{3}=65 \ldots 96$. These sequences define a grid

\footnotetext{
${ }^{1}$ For any pair of network configurations $(x, y) \in X \times X$, such that $s(y) \succeq s(x)$ holds, we say that configuration $y$ dominates configuration $x$.
}

Table 2 Demand ranges

\begin{tabular}{llllll}
\hline$R_{A}^{3}$ & $65-90$ & $R^{30}$ & $R^{31}$ & $R^{32}$ & $R^{33}$ \\
$R_{A}^{2}$ & $33-64$ & $R^{20}$ & $R^{21}$ & $R^{22}$ & $R^{23}$ \\
$R_{A}^{1}$ & $1-32$ & $R^{10}$ & $R^{11}$ & $R^{12}$ & $R^{13}$ \\
$R_{A}^{0}$ & 0 & $R^{00}$ & $R^{01}$ & $R^{02}$ & $R^{03}$ \\
& & 0 & $1-32$ & $33-64$ & $65-90$ \\
& & $R_{B}^{0}$ & $R_{B}^{1}$ & $R_{B}^{2}$ & $R_{B}^{3}$ \\
\hline
\end{tabular}

Table 3 Optimal compatible configurations

\begin{tabular}{llllll}
\hline$R_{A}^{3}$ & \multirow{2}{*}{$65-90$} & $(140)$ & $(170)$ & $(210)$ & $(240)$ \\
& & $x_{12}$ & $x_{13}$ & $x_{14}$ & $x_{15}$ \\
$R_{A}^{2}$ & $33-64$ & $(70)$ & $(140)$ & $(140)$ & $(210)$ \\
& & $x_{8}$ & $x_{9}$ & $x_{10}$ & $x_{11}$ \\
$R_{A}^{1}$ & \multirow{2}{*}{$1-32$} & $(70)$ & $(100)$ & $(140)$ & $(170)$ \\
& & $x_{4}$ & $x_{5}$ & $x_{6}$ & $x_{7}$ \\
$R_{A}^{0}$ & 0 & $(0)$ & $(70)$ & $(70)$ & $(140)$ \\
& & $x_{0}$ & $x_{1}$ & $x_{2}$ & $x_{3}$ \\
& & 0 & $1-32$ & $33-64$ & $65-90$ \\
& & $R_{B}^{0}$ & $R_{B}^{1}$ & $R_{B}^{2}$ & $R_{B}^{3}$ \\
\hline
\end{tabular}

of demand ranges $\mathfrak{R}=R_{A} \times R_{B}=\left\{R^{a b}: a \in 0 \ldots 3, b \in\right.$ $0 \ldots 3\}$ that constitute a partition of set of demands $\mathfrak{D}$ (see Table 2). Notice that example demand vectors $d_{I}, d_{I I}$, and $d_{I I I}$ fall, respectively, into ranges $R^{33}, R^{22}$, and $R^{11}$. Obviously, every but $R^{33}=\left(R_{A}^{3}, R_{B}^{3}\right)$ demand range in Table 2 has more than one compatible network configuration. To pave a way for a selection of the most appropriate one, for the sake of this example only, we introduce a simplistic cost function $c^{s}(x), x \in X$, that takes into account solely the cost of deployed optical fibres, i.e., defined as:

$c^{s}(x)=n^{H}(x) \zeta^{H}+n^{D}(x) \zeta^{D}, \quad x \in X$.

Constants $\zeta^{H}$ and $\zeta^{D}$ denote the cost of a single fibre in, respectively, $C O-D P$ and $D P-A P$ relations; to facilitate further analysis, values $\zeta^{H}=40$ and $\zeta^{D}=30$ are arbitrarily assumed. $^{2}$

Eventually, optimal compatible configurations of each range (the optimal costs are written in parentheses) obtained w.r.t. cost function (1) are shown in Table 3. Referring to the example from the very begin of this section, we can conclude that optimal compatible configurations for demand vectors $d_{I}, d_{I I}$, and $d_{I I I}$ (and thus, for ranges $R^{33}, R^{22}$, and $R^{11}$ ) are, respectively, $x_{15}, x_{10}$, and $x_{5}$.

The above conclusion holds true when an individual demand vector is considered. In the reality however it is

\footnotetext{
${ }^{2}$ Although being set arbitrarily, they reflect the proportion between cost of CO-DP and DP-AP fibres, provided the CO-DP component express also the cost of an OLT-card's port used.
} 
hardly the case-we observe not just a single but a sequence of demand vectors, each of them remaining present for a certain period of time. To cope with that issue we have to complement our formalism with new elements. We distinguish set $\mathcal{T}=\{0,1, \ldots, T\}$, of time periods (period $t=0$ denotes time before the actual network deployment start with, by the assumption, null demand vector and empty configurationwe consider the green-field deployment), that-depending on the dynamics of the market and policy of the operatormay correspond to either weeks, months, years, and so on. What is crucial, we assume that within a single period $t \in \mathcal{T}$, neither a demand vector nor a selected compatible network configuration changes. We represent a sequence of demand vectors that spans over the considered set of time periods by $\left\{d_{t}\right\}_{t \in \mathcal{T}}$, the sequence of demand ranges respective demand vectors fall into by $\left\{R_{t}\right\}_{t \in \mathcal{T}}$, and a sequence of network configurations by $\left\{x_{t}\right\}_{t \in \mathcal{T}}$. Any sequence of network configurations $\left\{x_{t}\right\}_{t \in \mathcal{T}}$, such that every element $x_{t}, t \in \mathcal{T}$, of the sequence is compatible with the corresponding demand vector $d_{t}$ (and thus, with demand range $R_{t}$ ), is called compatible to the sequence of demand vectors $\left\{d_{t}\right\}_{t \in \mathcal{T}}$ (and to the sequence of demand ranges $\left\{R_{t}\right\}_{t \in \mathcal{T}}$ ). Additionally, we introduce a set of period transitions $\mathcal{J}=\{(k, t): k \in$ $\mathcal{T} \backslash\{T\}, t \in \mathcal{T} \backslash\{0\}, t=k+1\}$, where each transition $j \in \mathcal{J}$, is an ordered pair $(k, t)$ of two consecutive time periods (distinguished as $k=a(j)$ and $t=z(j)$ ). For each transition $j \in \mathcal{J}$, we call a vector $r^{j}=\left(r_{\text {ins }}^{j}, r_{\text {ext }}^{j}\right.$ : $\left.r_{i n s}^{j}=x_{z(j)} \ominus x_{a(j)}, r_{e x t}^{j}=x_{a(j)} \ominus x_{z(j)}\right)$, the reconfiguration vector, with installation component $r_{i n s}^{j}$ and extraction component $r_{\text {ext }}^{j}$ (symbol $\ominus$ denotes here the subtraction operation modified in such a way that for any $(x, y) \in R^{N} \times R^{N}$, it gives vector $x \ominus y=\left(\max \left(x_{i}-y_{i}, 0\right): i \in 1 \ldots N\right)$ with every component non-negative). We do not restrict reconfigurations-both $r_{i n s}^{j}$ and $r_{e x t}^{j}$ components can attain any non-negative value, and thus, both equipment installation and extraction are admissible in every transition. Finally, we name vectors with null extraction and null both components as, in order, pure incremental reconfigurations and empty reconfigurations.

To designate the most cost-effective sequence of network configurations compatible to a given sequence of demand vectors one has to solve a multi-period optimization problem. The problem consists of a set of configuration-related constraints, which enforce compatibility of the whole selected sequence of configurations to the given sequence of demand vectors, and of a set of reconfiguration-related constraints and/or the objective function components, that aim at limiting the amount of equipment that can be exchanged in reconfigurations. Reconfiguration constraints and objective function components enforce that the problem does not fall into independently optimized pieces (what would lead to, unacceptable from the practical point of view, reconfiguration with the stub release - see, e.g., [25])

Notice that cost function (1) cannot be directly applied for evaluation of multi-period network deployment. To do so, one has to transform CAPEX expenditures related to the resource deployment into per-period resource usage OPEX-like expenditures; for the sake of this example-more detailed discussion is presented in Sect. 4-we estimate this per-period cost dividing the CAPEX by the number of periods $T$; finally, the multi-period version of our simplistic cost function takes the following form:

$$
\begin{aligned}
\bar{c}^{S}\left(\left\{x_{t}\right\}\right)= & \sum_{t \in \mathcal{T}}\left(n^{H}\left(x_{t}\right) \bar{\zeta}^{H}+n^{D}\left(x_{t}\right) \bar{\zeta}^{D}\right) \\
& +\sum_{j \in \mathcal{J}} C(j) .
\end{aligned}
$$

The first line of expression (2) is very similar to cost function (1) and represents the cost of every configuration of the sequence. It refers to constants $n^{H}\left(x_{t}\right)$ and $n^{D}\left(x_{t}\right)$ denoting the number of utilized fibres (in $C O-D P$ and $D P-A P$ relations) in particular time periods and to constants $\bar{\zeta}^{H}=\frac{40}{T}$ and $\bar{\zeta}^{D}=\frac{30}{T}$ representing the cost of a single-period usage of a single $C O-D P$ and $D P-A P$ fibre, respectively. The second line, of expression (2), is a novelty and expresses the cost of every reconfiguration.

Returning to our example-if neither reconfiguration cost (2) nor reconfiguration-related constrains are present, sequence $\left\{x_{0}, x_{5}, x_{10}, x_{15}\right\}$ is obviously optimal w.r.t. the multi-period cost function. If the reconfiguration cost starts doing matter, alternative sequences could be designated as, e.g., $\left\{x_{0}, x_{10}, x_{10}, x_{15}\right\}$ or even $\left\{x_{0}, x_{15}, x_{15}, x_{15}\right\}$, because they contain, respectively, one and two empty reconfigurations. Similar results can be obtained by enforcing that only pure incremental reconfigurations are admissible, which will exclude sequence $\left\{x_{0}, x_{5}, x_{10}, x_{15}\right\}$.

In our example, we assume that a sequence of demand vectors is given what allows us to formulate an optimization problem and to find the optimal sequence of network configurations. In the reality however, the deployment-after the network initial set-up-is driven by as a sequence of decisions problems; in every step, the future network evolution is decided, taking into account the current state and the nearfuture requirements (anticipated on the basis of historical data and the assumed model of the customer arrival process). Despite the differences, we are in the position that our approach is valuable-first, it introduces business and cost models suitable for multi-period analysis of FTTx network deployment cost; second, it gives a benchmark solution that can be used to evaluate the quality of methods applied in practice; and third it provides an opportunity to evaluate the 
influence of particular design decisions or restrictions onto cost and a trajectory of network evolution.

\section{Network model}

In this section, we introduce a model for the FTTx Optical Access Network (FTTx OAN) network, which we intend to exploit to formulate both a business model and the multiperiod optimization problem. The model is complemented with the definition of equipment catalog sets. The model considers OANs built upon the GPON standardization, in the architecture illustrated in Fig. 2 (we are in the position that the model is directly applicable to either PON, GPON, XGPON, and with minor modifications, also to WDM-(TDM)PON—see, e.g., [28] for comparison of PON technologies). According to the standardization (c.f. [19,21]) an FTTx OAN has a span from the service node interface (SNI) to the user network interfaces (UNIs), i.e., between the northbound interface of an OLT device and the southbound interface of an ONT. All ONTs connected to an FTTx OAN are partitioned into groups (of up to 128 devices) each one fed with an optical signal from a single port of an OLT device. The structure that distributes the signal to every ONT of the group is denoted (see Fig. 2) by optical distribution network (ODN) and delimited by $S / R$ and $R / S$ reference points.

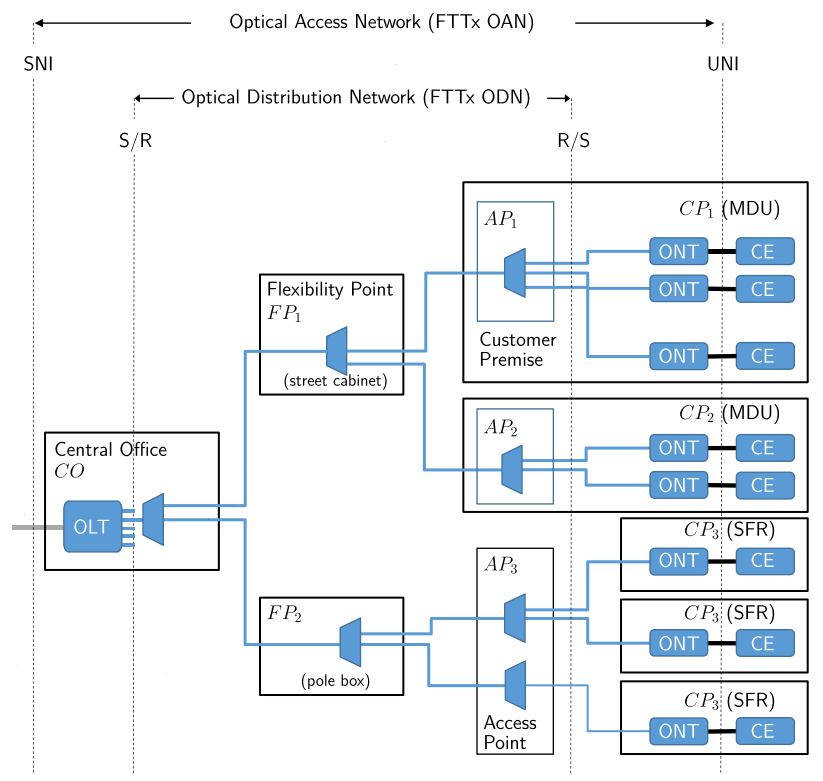

Fig. 2 Considered FTTx network architecture ( $A P$ access point, $C O$ central office, $C P$ customer premise, $F P$ flexibility point, $M D U$ multi family unit, $O A N$ optical access network, $O D N$ optical distribution network, $O L T$ optical line termination, $O N T$ optical network termination, SFR single family residential, SNI service node interface, UNI user network interface)

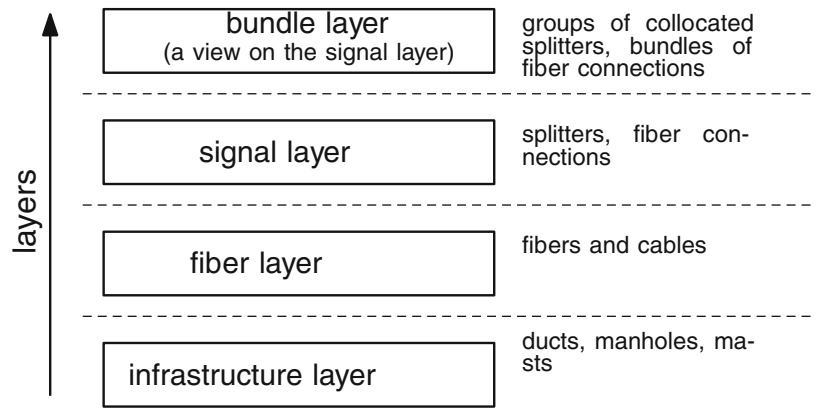

Fig. 3 Layers of our FTTx network model

Taking into account a position expressed by our industrial partners, we restrict our investigations to 3-tier ODNs that admit insertion of up to three splitters on the way between an OLT and an ONT. This can be considered as an extension of the model commonly referred to in the literature (e.g., $[8,28]$ ) that gives more flexibility in splitting paths by introducing additional splitters at the $\mathrm{CO}$ sites (disabling this extension is easy and would not influence our model). Locations where particular splitters can be installed are (see Fig. 2) the Central Office, dedicated distribution nodes (e.g., wells, street cabinets or pole mast boxes), and access nodes. Depending on the predominant type of buildings in the deployment area-multi dwelling units (MDU) or single-family residential (SFR)the access nodes are to be installed in customer premises or in dedicated outdoor compartments.

According to [20], we split the model into a hierarchy of layers. The splitting adheres to the client-server model where, for each pair of neighbor layers (in Fig. 3), the upper layer (the client) takes advantage of resources provided by the lower one (the server). In this model, each individual layer is described by means of a network of the layer specific nodes and links, hereafter referred to as the layer network.

\subsection{Infrastructure layer}

The infrastructure layer of the FTTx-OAN network (see Fig. 4) is the bottom layer of the model. It is characterized

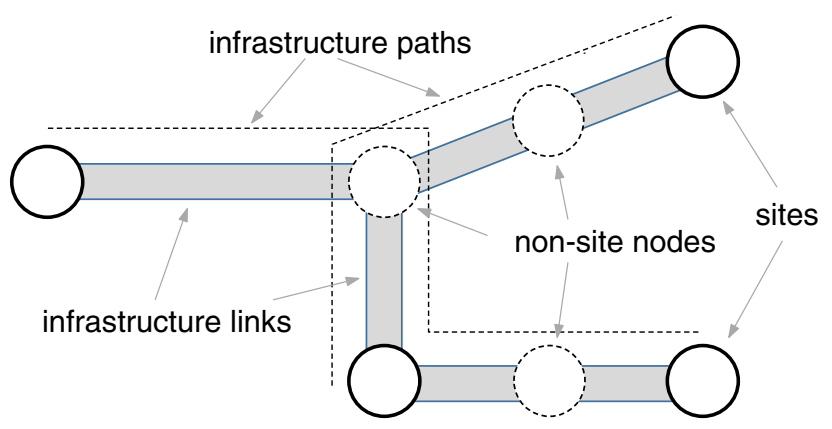

Fig. 4 Elements of infrastructure layer 
by FTTx-OAN infrastructure layer network $G^{I}=\left(\mathcal{N}^{I}, \mathcal{L}^{I}\right)$ with infrastructure nodes $\mathcal{N}^{I}$ (circles in the figure) representing, e.g., in-building compartments, manholes, masts or street cabinets, interconnected by infrastructure links $\mathcal{L}^{I} \subseteq$ $\mathcal{N}^{I} \times \mathcal{N}^{I}$ (edges connecting circle pairs) that stand for either trenches or overhead lines.

Within infrastructure nodes $\mathcal{N}^{I}$ we distinguish subset $\mathcal{N}^{I S} \subseteq \mathcal{N}^{I}$, of infrastructure sites (solid line circles) equipped to hold either active (OLT, ONT) or passive (optical splitters) devices. Set of infrastructure sites $\mathcal{N}^{I S}$ is further partitioned into central sites $\mathcal{N}^{I S C}$ (which is the singleton, i.e. $\mathcal{N}^{I S C} \equiv\left\{n^{I S C}\right\}$ ), distribution sites $\mathcal{N}^{I S D}$, and access sites $\mathcal{N}^{I S A}$, hereafter referred to as classes of infrastructure sites.

Let $\mathcal{P}^{I} \subseteq 2^{\mathcal{L}^{I}}$, denote a set of paths in the infrastructure layer and let $i i_{\text {pathNodes }}: \quad \mathcal{P}^{\mathcal{I}} \Rightarrow 2^{\mathcal{N}^{\mathcal{I}} \times \mathcal{N}^{\mathcal{I}}}$ designate a function that returns a pair of end nodes of path $p \in \mathcal{P}^{I}$. We distinguish a subset $\mathcal{P}^{I S} \subseteq \mathcal{P}^{I}$, of infrastructure paths, hereafter referred to as infrastructure trails, having both their end nodes in the set of infrastructure sites, i.e., $t \in \mathcal{P}^{I S} \Rightarrow$ $i i_{\text {pathNodes }}(t) \in 2^{N^{I S} \times \mathcal{N}^{I S}}$.

\subsection{Fibre layer}

The fibre layer network (see Fig. 5) is modeled by graph $\mathcal{G}^{F}=\left(\mathcal{N}^{F}, \mathcal{L}^{F}\right.$ ) with set of fibre nodes $\mathcal{N}^{F}$ (optical splitters) and set of fibre links $\mathcal{L}^{F}$ (segments of a fibre enclosed in cables).

Let $\mathcal{P}^{F} \subseteq 2^{\mathcal{L}^{\mathcal{F}}}$, designate a set of paths in the fibre layer, hereafter referred to as fibre paths, where each represents a continuous sequence of fibre links joint by means of splices or switched in optical distribution frames (ODF). An infrastructure trail $t \in P^{I S}$, can accommodate a group of fibre paths distinguished by function if fiber Paths $: \mathcal{P}^{I S} \Rightarrow 2^{\mathcal{P}^{F}}$ that interconnects optical splitters installed in trail's end infrastructure sites. Fibre paths are further split into trunk fibre

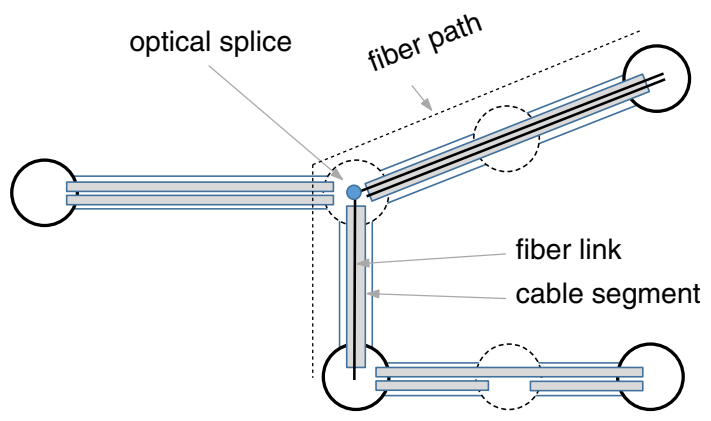

Fig. 5 Elements of fibre layer

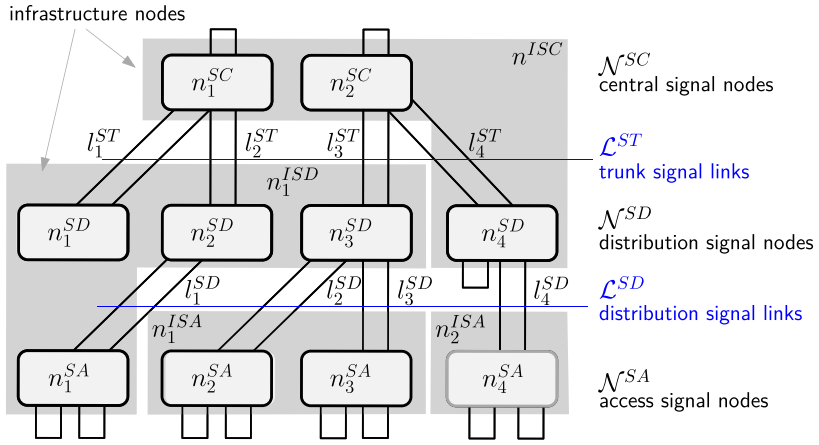

Fig. 6 Elements of signal layer

paths $\mathcal{P}^{F T}$, which support trunk bundle links, and distribution fibre paths $P^{F D}$ supporting distribution bundle links. ${ }^{3}$

\subsection{Signal layer}

The FTTx-OAN signal layer network (see Fig. 6) plays the crucial role in our network model and provides bidirectional signal connections between ports of OLT and ONT devices, i.e., between $S / R$ and $R / S$ reference points of the architecture presented in Fig. 2. We characterize this layer network using graph $\mathcal{G}^{S}=\left(\mathcal{N}^{S}, \mathcal{L}^{S}\right.$ ) with set of nodes $\mathcal{N}^{S}$ (represented by rounded rectangles in the figure) and set of links $\mathcal{L}^{S}$, where signal node $n \in \mathcal{N}^{S}$, stands for an optical splitter and signal link $l \in \mathcal{L}^{S}$, represents exactly one fibre path between a pair of connected splitters.

As already mentioned, we consider the architecture, where each signal connection goes through exactly three signal nodes (splitters) ${ }^{4}$ referred to-counting from the OLT's side-as central signal node, distribution signal node, and access signal node; due to a splitter belonging to exactly one FTTx-ODN network, this rule partitions set $\mathcal{N}^{S}$ into, respectively, $\mathcal{N}^{S C}, \mathcal{N}^{S D}$, and $\mathcal{N}^{S A}$ subsets referred to as classes of signal nodes.

There are two distinguished classes of signal links: trunk signal links $\mathcal{L}^{S T} \subseteq \mathcal{L}^{S}$, between the central and distribution signal nodes and, distribution signal links $\mathcal{L}^{S D} \subseteq \mathcal{L}^{S}$, between distribution and access signal nodes. These two classes constitute the partition of set $\mathcal{L}^{S}$.

To facilitate further analysis of inter-layer dependencies, we introduce function $s i_{\text {site }}: \mathcal{N}^{S} \rightarrow \mathcal{N}^{I S}$ that distinguishes infrastructure site $i \in \mathcal{N}^{I S}$, that hosts signal node $s \in \mathcal{N}^{S}$ (exemplary layout of infrastructure nodes that could host pre-

\footnotetext{
${ }^{3}$ To complete the description: a fibre $\operatorname{link} l \in \mathcal{L}^{\mathcal{F}}$, is in turn a continuous segment of an optical fibre that (together with other fibre segments) resides within a single cable link. Finally, the optical cable link is a continuous segment of optical cable deployed in an infrastructure path consisting of one or more infrastructure links.

4 To support this assumption, we introduce an artificial null splitter with 1:1 split ratio and zero attenuation.
} 
sented signal nodes is represented in Fig. 6 by grayed-out polygons). The following cases are admissible:

- signal access node $s \in \mathcal{N}^{S A}$, can be located in an infrastructure site of any class, i.e., $s i_{\text {site }}(s) \in \mathcal{N}^{I S}$,

- signal distribution node $s \in \mathcal{N}^{S D}$, can be located either in one of distribution infrastructure sites or the central infrastructure site, i.e., $\operatorname{si}_{\text {site }}(s) \in \mathcal{N}^{I S D} \cup\left\{n^{I S C}\right\}$,

- signal central node $s \in \mathcal{N}^{S C}$, can be located solely in the central infrastructure node, i.e., $s i_{\text {site }}(s) \equiv n^{I S C}$.

We attribute each access signal node $s \in \mathcal{N}^{S A}$, with a number $h^{S t s}$ denoting the number of ONTs connected to the node at $R / S$ reference point, i.e., the signal demand of this node in time period $t \in \mathcal{T}$.

\subsection{Bundle layer}

As the signal layer model identifies every individual element necessary to provide signal network connections between OLT and ONT devices, its direct application would lead to unacceptably large optimization problem instances; thus, we decided to introduce the aggregated model, called the bundle layer model, that considers bundles (groups) of signal nodes and signal links instead of individual ones. The bundle layer model is what we exploit in the remaining sections.

The considered bundle layer model (see Fig. 7) consists of directed graph $\mathcal{G}^{B}=\left(\mathcal{N}^{B}, \mathcal{L}^{B}\right)$ with set of bundle nodes $\mathcal{N}^{B}$ and set of bundle links $\mathcal{L}^{B}$. Graph $\mathcal{G}^{B}$ constitutes a contraction of graph $\mathcal{G}^{S}$ of the signal layer. Thus each bundle node $b \in \mathcal{N}^{B}$, represents a subset of signal nodes (distinguished by function $b s_{\text {nodes }}: \mathcal{N}^{B} \rightarrow 2^{\mathcal{N}^{S}}$ ) such that:

- all of them are located in the same infrastructure site, i.e., $\exists i \in \mathcal{N}^{I S}, \forall s \in b s_{\text {nodes }}(b) \Rightarrow s i_{\text {site }}(s)=i$,

- all of them belong to the same class of the signal nodes, i.e., $\forall s \in b s_{\text {nodes }}(b)$ exactly one of: $s \in \mathcal{N}^{S C}, s \in \mathcal{N}^{S D}$ or $s \in \mathcal{N}^{S A}$ holds true.

We emphasize that the above assignment is exhaustive, i.e., within a single infrastructure site there is at most one bundle node of each particular class. According to the latter requirement, we introduce the partitioning of bundle nodes $\mathcal{N}^{B}$ into central bundle nodes $\mathcal{N}^{B C}$ (which is a singleton, i.e., $\mathcal{N}^{B C} \equiv\left\{n^{B C}\right\}$ ), distribution bundle nodes $\mathcal{N}^{B D}$, and access bundle nodes $\mathcal{N}^{B A}$, hereafter referred to as classes of bundle nodes. Similarly to the signal layer case, each bundle node of the access class $b \in \mathcal{N}^{B A}$ representing subset $\mathcal{N}^{S B}=b s_{\text {nodes }}(b)$ of access signal nodes, is attributed with signal demand $h^{B t b}=\sum_{s \in \mathcal{N}^{\mathcal{S B}}} h^{\text {Sts }}$, i.e., its signal demand in period $t \in \mathcal{T}$, is equal to the sum of demands of these signal nodes.
Bundle link $l \in \mathcal{L}^{B}$, represents in turn every signal link connecting signal nodes aggregated to the end bundle nodes of this bundle link (we use function $b s_{\text {links }}: \mathcal{L}^{B} \rightarrow 2^{\mathcal{L}^{S}}$ that distinguish such set of signal links). Certainly, every signal link aggregated into a bundle link belongs to the same signal link class. Taking advantage of this observation, we partition also bundle links $\mathcal{L}^{B}$ into trunk bundle links $\mathcal{L}^{B T}$ between the central and a distribution bundle nodes and distribution bundle links $\mathcal{L}^{B D}$ between a distribution and an access bundle node. We refer to this partitioning as bundle link classes. We assume that all fibre paths that support a given bundle link use the same infrastructure trail.

\subsection{Example of mapping between signal and bundle layers}

Figures 6 and 7 show configurations of signal and bundle layers that are mutually compatible. The signal layer model (see Fig. 6) comprises signal nodes and signal links of every particular class. Nodes co-located within the same infrastructure sites are enclosed within gray polygons. The bundle counterpart of this node is illustrated in Fig. 7. According to rules set in Sect. 3.4, a bundle node in a given infrastructural site aggregates every signal node of the same class located in this site and, within an infrastructure site, there is at most one bundle node of each particular class. Thus, in the example, the following contraction rules are applied:

- for central bundle nodes:

$$
\text { - bs nodes }\left(n^{B C}\right)=\left\{n_{1}^{S C}, n_{2}^{S C}\right\},
$$

- for distribution bundle nodes:

$$
\begin{aligned}
& -b s_{\text {nodes }}\left(n_{1}^{B D}\right)=\left\{n_{1}^{S D}, n_{2}^{S D}, n_{3}^{S D}\right\}, \\
& -b s_{\text {nodes }}\left(n_{2}^{S D}\right)=\left\{n_{4}^{S D}\right\},
\end{aligned}
$$

- for access bundle nodes:

$$
\begin{aligned}
& -b s_{\text {nodes }}\left(n_{1}^{B A}\right)=\left\{n_{1}^{S A}\right\}, \\
& -b s_{\text {nodes }}\left(n_{2}^{B A}\right)=\left\{n_{2}^{S A}, n_{3}^{S A}\right\}, \\
& -b s_{\text {nodes }}\left(n_{3}^{B A}\right)=\left\{n_{4}^{S A}\right\},
\end{aligned}
$$

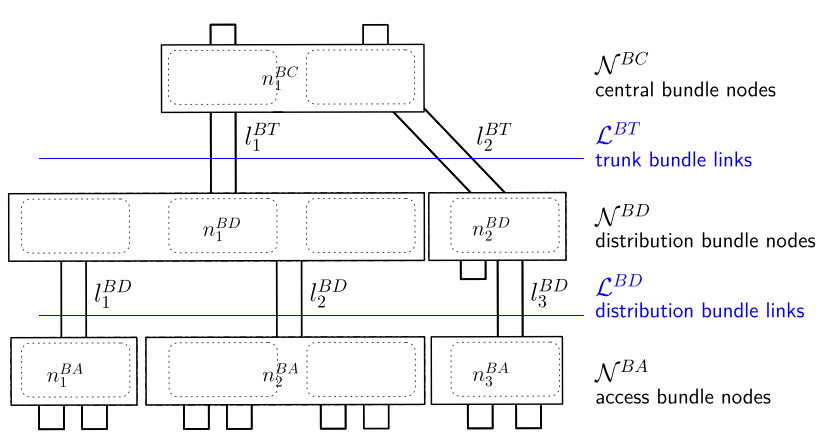

Fig. 7 Elements of bundle layer 
- for trunk bundle links:

$$
\begin{aligned}
& -b s_{\text {links }}\left(l_{1}^{B T}\right)=\left\{l_{1}^{S T}, l_{2}^{S T}, l_{3}^{S T}\right\}, \\
& -b s_{\text {links }}\left(l_{2}^{B T}\right)=\left\{l_{4}^{S T}\right\},
\end{aligned}
$$

- for distribution bundle links:

$$
\begin{aligned}
& -b s_{\text {links }}\left(l_{1}^{B D}\right)=\left\{l_{1}^{S D}\right\}, \\
& -b s_{\text {links }}\left(l_{2}^{B D}\right)=\left\{l_{2}^{S D}, l_{3}^{S D}\right\}, \\
& -b s_{\text {links }}\left(l_{3}^{B D}\right)=\left\{l_{4}^{S D}\right\} .
\end{aligned}
$$

\subsection{Equipment catalog sets}

This subsection completes the network model with catalog sets defining types of equipment admissible for installation at infrastructure sites and links. Every catalog set is denoted by $C$ with a lowercase upper index; the same lowercase index is used for distinguishing properties of an instance of a particular type. Parameters common to every type, like cost (i.e., the capital expenditure-CAPEX), period lease cost or capacity, are denoted by lowercase letters $c, \varsigma$, and $\eta$ with appropriate upper indices.

\subsubsection{OLT cards}

We assume that there is only one type of OLT cards; each card of this type is characterized by its cost $c^{C}$, number $\eta^{C}$ of ports it holds, and transmission signal power $p^{C}$ of its ports.

\subsubsection{OLT devices}

We assume that there is only one type of OLT devices; each OLT device of this type is characterized by its $\operatorname{cost} c^{O}$, weight $w^{O}$ (i.e., the amount of OLT capacity $\eta^{f o}$ it requires in the hosting site-see 3.6.5), maximum number $\eta^{O}$ of OLT cards it can hold.

\subsubsection{Optical splitters}

Set $\mathcal{C}^{r}$ identifies admissible types of optical splitters. Each particular type of splitter $r \in \mathcal{C}^{r}$, is characterized by its cost $c^{r}$, split ratio $\eta^{r}$, and attenuation $a^{r}$ a splitter introduces into a signal network connection. Set $C^{r}$ is complemented by an artificial null splitter $r^{1}$ with $\eta^{r^{1}}=1, a^{r^{1}}=0$, and $c^{r^{1}}=0$. The null splitter facilitates modeling by preserving structural homogeneity of signal network connections.

\subsubsection{Admissible splitter combinations}

Set of admissible triples of splitter types $\mathcal{C}^{3 r} \subseteq \mathcal{C}^{r} \times \mathcal{C}^{r} \times \mathcal{C}^{r}$ identifies applicable splitting patterns. This allows for reduction of the number of considered splitting combination, and thus, may lead to shortening of the computation time. ${ }^{5}$ To facilitate the formulation, we additionally introduce set of admissible splitter type pairs $\mathcal{C}^{2 r} \subseteq \mathcal{C}^{r} \times \mathcal{C}^{r}$; certainly, sets $\mathcal{C}^{3 r}$ and $\mathcal{C}^{2 r}$ must be consistent, i.e., $\mathcal{C}^{2 r}=\{(r, s)$ : $\left.\exists(t, u, v) \in \mathcal{C}^{3 r}, r=t, s=u\right\}$.

\subsubsection{Sites}

Each site type $f \in C^{f}$, represents a possible site arrangement, e.g., dedicated building, street cabinet, pole box, etc.; it is characterized by its $\operatorname{cost} c^{f}$ and two capacity parameterscabinet capacity $\eta^{f e}$ and OLT capacity $\eta^{f o}$ - that define the maximum total weight of, respectively, cabinets and OLT devices a site of the particular type can host.

\subsubsection{Hardware cabinets}

Equipment deployed within a site is installed in one or more hardware cabinets (equipment racks). Each cabinet type $e \in C^{e}$, is characterized by its weight $w^{e}$ (i.e., the amount of cabinet space $\eta^{f e}$ it consumes within a hosting site), $\operatorname{cost} c^{e}$, and the maximum number of splitter ports $\eta^{e}$ it can accommodate.

\section{Business and cost model}

This section introduces a cost model we use for analyzing the multi-period FTTx network operation.

We consider the NP business entity (playing the network provider role) that organizes and operates an access network delivering the broadband access service to a set of customers. Traditionally, the NP-entity would adhere to the vertically integrated (or stovepipe) model where a single company owns every element of the communication value-chainfrom the passive infrastructure, through network resources, up to service delivery to individual customers. Nowadays, this gradually ceases to be the case; the reason for this is two-fold-there are business drives that force companies to seek ways to share huge investment costs (see $[29,30]$ ) as well as the regulatory drives at either municipal, state or international level (cf. [7,14,24]) that tend to enforce open and fair access to the communication resources. What we observe in the broadband access business is a shift towards the infrastructure provider-service provider decoupling paradigm, being already applied for years in such industries like the airline industry (where airport operators, i.e., infrastructure providers, are separated from the air-

\footnotetext{
5 Introduction of this set is an outcome of discussions conducted with our industrial partners. They are in the position that it is a common trend to limit the number of patterns used in the network to preserve homogeneity and to reduce operational costs.
} 
lines, i.e., service providers) or the railways industry (with separated railway network operators and train operator companies). The same paradigm, relatively recently, found its application in communication network virtualization, where many independent virtual networks are created on the basis of resources (network slices) built from elements taken from the shared infrastructure or data center virtualization, where the data center operator provides (as the infrastructure provider) IaaS (Infrastructure as a Service) services for a large number of customers.

In our model, we introduce two additional business entities that play the infrastructure provider role w.r.t. our NP. First, according to [7], we identify the passive infrastructure provider (PIP) entity that holds and sells access to infrastructure resources (duct space, optical cables, optical fibres, etc.). Second, we introduce the equipment provider (EP) entity that has in its disposal either passive (distribution frames, optical splitters) and active (OLT devices and OLT cards) network equipment and leases the equipment to the NP, on its demand (the EP role has not be identified in [7]). We assume that NP can change configuration of its network (either install or extract resources and equipment) on period-to-period basis to adapt to varying number of customers, paying for the resources and equipment actually used. In our model, the NP-entity pays also for transition between configurations of consecutive periods-that comprises cost of site-surveys and cost of the actual installation/extraction of equipment.

Observe that the business entities identified in the model may represent organizational units/divisions of either different companies or of a single company. The former case is obvious, as this corresponds to ordinary market exchange of products between autonomous companies. The latter case corresponds to intra-company transfer of division's products (see [4]); similar approach is commonly deployed by many companies for the sake of accounting management procedures that are to judge on economical efficiency of their particular divisions. Observe that mixed cases are also admissible - e.g., PIP-type division of company A can lease infrastructure resources simultaneously to NP-division of the same company and to NP-divisions of other companies.

This section is organized as follows. Section 4.1 introduces business-oriented stratification of access network resources. Section 4.2 formalizes business roles and identifies a number business architectures, i.e., ways these roles can be assigned to different business players. Section 4.3 shows a draft of the depreciation approach necessary to account CAPEX expenditures as OPEX expenses. Finally, Sect. 4.4 shows our cost model.

\subsection{Business layers}

From the business perspective, as illustrated in Fig. 8, resources of the FTTx network are split into hierarchy of business layers (c.f. $[7,13,14])$. The lowest passive infrastructure layer (corresponding to the infrastructure and fibre layers of the model introduced in Sect. 3) comprises all passive elements of the network including cable placeholders of every kind (trenches, ducts, sewage systems, and overhead lines), equipment placeholders (central office buildings, street cabinets, manholes, and pole masts), optical cables, individual optical fibres (dark fibres), and finally, individual wavelengths within a each fibre. The next active network layer (corresponding to the signal and bundle layers of our network model-see Sect. 3) embraces hardware (e.g., OLT devices, optical splitters, ONU/ONT devices) and software components (IT tools) that connect and orchestrate resources exposed by the passive network layer and provides data transport (broadband IP or Ethernet access) between SNI and UNI reference points. The retail service layer consumes the wholesale transport service and provides retail services offered to the end-users (these are common triple-play services like broadband internet access, voice communication, TV-broadcasting, and Video-on-Demand) complemented with operator/location specific services (like e-health, elderly care, video-conferencing, entertainment, teleworking, e-gov, e-education, e-commerce, smart monitoring, internet of things, cloud computing, and so on-cf. [14]). Finally, the end-user layer (not presented in the figure) represents every subscriber of every kind-domestic users, small companies (e.g., SOHO), large companies, and institutional (government, municipal), police, fire guard, medical, etc.

\subsection{Business architectures}

The layered architecture paves a way for identification of roles played by business entities in the context of FTTx networks (see Fig. 8). These are:

- Passive Infrastructure Provider (PIP) that posses (a part of) the passive infrastructure and provides (at the $I-N$ business boundary—see Fig. 8) one or more wholesale clients with an access to this infrastructure,

- Network Provider (NP) that consumes infrastructural elements obtained from a set of PIPs, orchestrates them to build and operate an active network, and provides the wholesale transport service to communication clients (3G/4G operators, cable TV operators, banks, large enterprises, the public sector, etc.—see [13]) as well as a set of retail service providers,

- Retail Service Provider (SP) that consumes wholesale data transport services obtained from a set of NP; provides retail services to individual subscribers.

In Fig. 8, two business reference points are also displayed, i.e., points of the interaction between pairs of roles. Both 


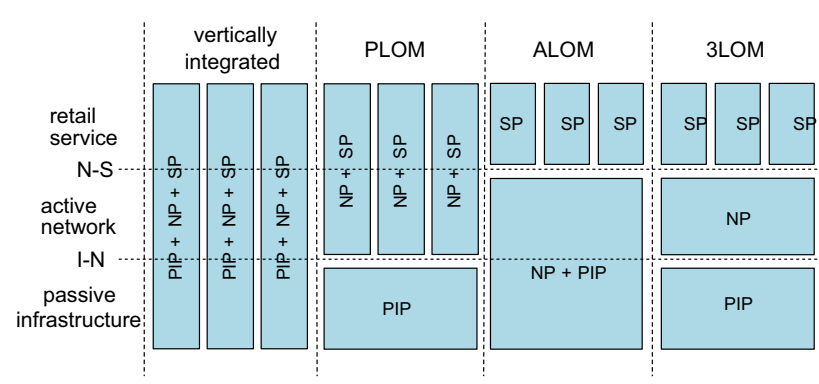

Fig. 8 Business modes

infrastructure to network $(I-N)$ point and network to service (N-S) point are of the customer to provider type, where the upper role buys products, either the resources or services, exposed by the lower role.

Traditionally, every of these roles is played, in so called vertically integrated (or stovepipe) model, by a single business entity (the leftmost case in Fig. 8). Nowadays, this gradually - as argued at the begining of this section-ceases to be the case. Business entities cooperate in different configurations and at various levels of the architecture; a couple of distinguished common architectures-referred to, respectively, as passive layer open model (PLOM), active layer open model (ALOM), and 3-layer open model (3LOM)are illustrated in respective subsequent parts of Fig. 8.

Depending on the business architecture, a reference point may become to be either internal or external when existing between respectively roles played by the same or different business entities. The external points (business interfaces) represent the actual selling of products between companies. The internal points in turn can be used in management accounting to identify and to value cost objects produced, processed, and exchanged between parts of a single company.

\subsection{PIP role cost accounting}

We consider the PIP business entity playing the passive infrastructure provider role that organizes and operates communication infrastructure, which can support one or more access networks. Without losing generality, ${ }^{6}$ we assume for a while that the entity owns all the resources comprising the infrastructure. The service the entity offers is leasing of infrastructure resources for a number of consecutive time periods. The price for the leasing is calculated as follows. First, the entity estimates its per-period total cost of ownership (TCO) on the basis of known per-period OPEX expenditures (related to salaries, insurance, energy consumption, etc.) and per-period cost of resource deprecation (to

\footnotetext{
6 The PIP entity can rent a part of its infrastructure from a number of other PIPs-e.g., duct space, optical cables, individual fibers installed by energy and water distribution companies.
}

account CAPEX expenditures). Second, it allocates the TCO cost to every resource is offers for lease, possibly adds its profit margin, and thus, receives the required per-period lease prices.

To imagine how depreciation (sometimes referred to also as amortization) can be computed consider set $\mathcal{A}^{e}$ of tangible assets owned by the PIP entity. Each asset $a \in \mathcal{A}^{e}$, is attributed with capital expenditure (CAPEX), denoted by $c(a)$, a PIP-entity had to spend for its deployment (duct preparation, cable rollout) or procurement (OLT, OLT card, splitter, cable segment). Asset $a \in \mathcal{A}^{e}$, has also defined the useful lifetime $l(a)$, expressed in the number of time periods (as introduced in Sect. 2), optionally, the salvage value $s(a)$ that defines a price the asset can be sold for after its useful lifetime has expired. The capital expenditure $c(a)$ is converted to in-period depreciation $\zeta^{t}(a), t \in 1,2, \ldots, l(a)$, in such a way that for each asset the following equation holds:

$\sum_{t \in 1,2, \ldots, l(a)} \zeta^{t}(a)=c(a)-s(a), \quad a \in \mathcal{A}^{e}$

In our experiments, we apply the linear deprecation model, where depreciation in every time period $t \in 1,2, \ldots, l(a)$ is constant, i.e., $\zeta^{t}(a)=\zeta(a)$ and estimated with the formula $\zeta(a)=\frac{c(a)-s(a)}{l(a)}$.

Let $\mathcal{P}^{e}, e \in \mathcal{E}$, denote a set of resources the PIP entity offers for leasing. The PIP-entity can apply a couple of known methods, e.g., long run incremental cost (LRIC) or fully distributed cost approach (FDCA) [11] (also referred to as fully allocated cost in [30]), to allocate the TCO cost to these resources. In our investigations, we selected the most obvious FDCA method where costs allocated to every resource sum up to exactly the total TCO. Depending on the business context, e.g., resources are leased to divisions of the same or different companies, the PIP-entity can add some profit margin to the cost allocated to the leased resource to receive its lease price (we denote this by $\zeta(p), p \in \mathcal{P}^{e}$ ).

\subsection{NP-role cost model}

In this subsection, we introduce a cost model for the NP role; the model is necessary to support the formulation of the multiperiod optimization problem in Sect. 5.

Consider the architecture presented in Fig. 9 that complements the architecture introduced in Sect. 4.2 with equipment provider role (EP) and the associated $E-N$ business reference point. The raison d'etre of the NE role is to support a set of (one or more) NPs with a possibility to lease the active (OLT, OLT cards) or passive (optical splitters) equipment. Having such a possibility, an NP role can install/extract the equipment on period-to-period basis paying in each period for the equipment actually installed in its network. The example justification of such model is the business entity that performs 


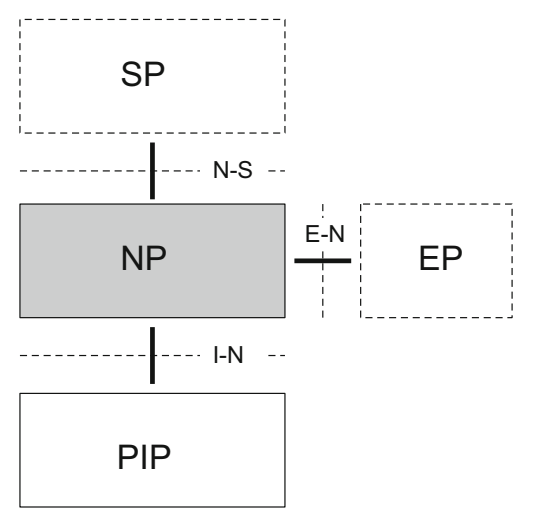

Fig. 9 Business roles considered in the cost model

the NP role simultaneously against many FTTx networksthe entity is free to move equipment (thus the equipment related cost) from one network to another. Certainly, in this example, the entity plays both NP and NE roles.

Finally, in our model, there are three reference points:

- $I-N$ that represents interactions between the PIP and NP roles; the infrastructure provider (PIP) grants there, on period-to-period basis, the access to the space for installation of equipment in infrastructure sites (cf. Sect. 3.1) and the access to fibre paths (see Sect. 3.2) between infrastructure sites,

- $E-N$ that represents interactions between the EP and NP roles; the equipment provider (EP) provides there, on period-to-period basis, passive (optical splitters of different split ratio) and active (OLT devices, OLT cards) equipment,

$-N-S$ that represents interactions between the NP and SP roles; the network provider (NP) provides there a set of optical connections (between $\mathrm{S} / \mathrm{R}$ and $\mathrm{R} / \mathrm{S}$ interfaces) as required by the SP role.

We consider costs associated with the NP role. According to the model introduced in the previous sections, we identify three categories of cost-we call them according to the business interface they are presented at $-I-N$ cost, $E-N$ cost, and $N P$ cost (i.e., the cost generated by the NP role itself). ${ }^{7}$

\subsubsection{E-N cost parameters}

- one-period installation of an OLT device; the associated one-period charge is denoted by $\zeta^{O}$,

\footnotetext{
7 We do not consider NP's income. This is due to the assumption that $\mathrm{NP}$ is obliged to connect every customer that wants a connection in every time period (we also assume that NP does not sell dark fibres or optical signals at $N-S$ business interface).
}

- one-period installation of an optical splitter; the associated one-period charge depends on the splitter type $r \in \mathcal{C}^{r}$ and is denoted by $\zeta^{r}$,

- one-period installation of an OLT card; as we assumed there is just one OLT card type, we denote the associated charge by $\zeta^{C}$.

\subsubsection{I-N cost parameters}

- one-period access to trunk fibre path $p^{F T} \in \mathcal{P}^{F T}$, that connects the central bundle node $n^{B C}$ (see Sect. 3.4) with a distribution bundle node $d \in \mathcal{N}^{B D}$; the associated one-period charge depends on the path and is denoted by $\zeta_{b}^{F T}, b \in \mathcal{N}^{B D}$,

- one-period access to distribution fibre path $p^{F D} \in \mathcal{P}^{F D}$, between distribution bundle node $d \in \mathcal{N}^{B D}$, and access bundle node $a \in \mathcal{N}^{B A}$; the associated one-period charge depends on the path and is denoted by $\zeta_{a}^{F D}, a \in \mathcal{N}^{B A}$,

- one-period access to one splitter port installation cabinet space in either central, distribution or access bundle node $n \in \mathcal{N}^{B C} \cup \mathcal{N}^{B D} \cup \mathcal{N}^{B A}$; the associated one periodcharge depends on node, it is denoted by $\zeta_{n}^{R}, n \in \mathcal{N}^{B}$; installation of $1: m$ splitter costs $(m+1) * \zeta_{n}^{R}$,

- one-period installation of one OLT port at the central infrastructure site; the associated one-period charge is denoted by $\zeta_{\text {port }}^{O}$.

\subsubsection{NP cost parameters}

- Site survey cost,

- splitter installation/extraction cost,

- OLT installation/extraction cost,

- OLT card installation/extraction cost.

\subsubsection{N-S cost parameters}

- One period delivery of an optical signal to a specified ONT device.

\section{Optimization problem}

Generally speaking we consider an optimization problem that aims at minimizing the total expenditures of a business entity playing the Network Provider (NP) role (see Sect. 4.2) during the whole network lifetime. The formulation exploits concepts and notions from the model introduced in Sect. 3, especially, the bundle layer model from Sect. 3.4. The presented approach takes its roots from the formulation of a single-period design problem introduced in [23] that enhances optimization platform presented in [35]. 


\subsection{Global formulation}

Consider a single time period $t \in \mathcal{T}$, (see Sect. 2). Let $x^{a t w} \in \mathbb{Z}_{+}$, denote an integer variable specifying the number of optical splitters of type $w \in \mathcal{C}^{r}$, installed in access bundle node $a \in \mathcal{N}^{B A}$, that in time period $t$ must be fed (through splitters of distribution and central bundle nodes) with an optical signal from any OLT port. Such connected splitters we hereafter call real splitters. Complementarily, let $\hat{x}^{a t w} \in \mathbb{Z}_{+}$, denote a similar integer variable specifying the number of splitters that are installed in time period $t$, but not necessarily connected in this period to OLT ports-we refer to them as virtual splitters. ${ }^{8}$ Continuing with the same naming scheme, we introduce pairs $\left(x^{d t s}, \hat{x}^{d t s}\right)$ and $\left(x^{C t r}, \hat{x}^{C t r}\right)$ of integer variables representing, respectively, the number of real and virtual splitters of type $s \in \mathcal{C}^{r}$, installed at period $t \in \mathcal{T}$, in every distribution bundle node $d \in \mathcal{N}^{B D}$, and the number of real and virtual splitters of type $r \in \mathcal{C}^{r}$, installed at period $t \in \mathcal{T}$, in the central bundle node $n^{B C}$. Finally, let $y^{C t}$ and $z^{C t}$ denote, in order, a variable representing the number of OLT cards and the number of OLT devices installed at period $t$.

Taking advantage of these variables, for each time period $t \in \mathcal{T}$, we introduce the following vectors describing equipment remaining installed in the network during this time period:

$\mathbf{x}^{\mathbf{A t}}=\left(x^{a t w}: a \in \mathcal{N}^{B A}, w \in \mathcal{C}^{r}\right)$,

$\hat{\mathbf{x}}^{\mathbf{A t}}=\left(\hat{x}^{a t w}: a \in \mathcal{N}^{B A}, w \in \mathcal{C}^{r}\right)$,

$\mathbf{x}^{\mathbf{D t}}=\left(x^{d t s}: d \in \mathcal{N}^{B D}, s \in \mathcal{C}^{r}\right)$,

$\hat{\mathbf{x}}^{\mathbf{D t}}=\left(\hat{x}^{d t s}: d \in \mathcal{N}^{B D}, s \in \mathcal{C}^{r}\right)$,

$\mathbf{x}^{\mathbf{C t}}=\left(x^{C t r}: r \in \mathcal{C}^{r}\right)$,

$\hat{\mathbf{x}}^{\mathbf{C t}}=\left(\hat{x}^{C t r}: r \in \mathcal{C}^{r}\right)$.

Then we introduce vector in the form (5) and refer to it as the configuration of the FTTx-OAN network in time period $t \in \mathcal{T}$.

$\mathbf{X}^{\mathbf{t}}=\left(\mathbf{x}^{\mathbf{A t}}, \hat{\mathbf{x}}^{\mathbf{A t}}, \mathbf{x}^{\mathbf{D t}}, \hat{\mathbf{x}}^{\mathbf{D t}}, \mathbf{x}^{\mathbf{C t}}, \hat{\mathbf{x}}^{\mathbf{C t}}, y^{C t}, z^{C t}\right)$.

Let $\mathscr{X}^{\mathbf{t}}$ denote the set of all configurations $\mathbf{X}^{\mathbf{t}}$ that satisfy every intra-period constraint of period $t \in \mathcal{T}$ (i.e., that are compatible - see Sect. 2-with a demand vector $d_{t}$ ); hereafter we refer to this set as an intra-period feasible set of period $t$ (constraints defining this set are described in detail in Sect. 5.2).

In the next step, consider a period transition $j \in \mathcal{J}$, between periods $k$ and $t$, i.e., $j=(k, t)(\mathcal{J}$ denotes a set of period transitions-see Sect. 1). Pair of variables

\footnotetext{
8 Virtual splitters can be installed in reserve to minimize the number of necessary site surveys and, as a result, operational costs of the NP.
}

( $x_{\text {ins }}^{a j w} \in \mathbb{R}_{+}, \hat{x}_{\text {ext }}^{a j w} \in \mathbb{R}_{+}$) counts the number of splitters of type $w \in \mathcal{C}^{r}$, which need to be installed/extracted from access bundle node $a \in \mathcal{N}^{B A}$, at the transition $j \in \mathcal{J}$; thus, expressions $x_{i n s}^{a j w}=\max \left(0, x^{a t w}+\hat{x}^{a t w}-x^{a k w}-\hat{x}^{a k w}\right)$ and $x_{\text {ext }}^{a j w}=\max \left(0, x^{a k w}+\hat{x}^{a k w}-x^{a t w}-\hat{x}^{a t w}\right)$ hold true. In the similar way, we introduce pairs $\left(x_{i n s}^{d j s}, x_{\text {ext }}^{d j s}\right), d \in$ $\mathcal{N}^{B D}, s \in \mathcal{C}^{r}$ and $\left(x_{i n s}^{C j r}, x_{\text {ext }}^{C j r}\right), c=n^{B C}, r \in \mathcal{C}^{r}$ that represent, respectively, the number of splitters of type $s \in \mathcal{C}^{r}$, installed/extracted from distribution bundle node $d \in \mathcal{N}^{B D}$, and the number of splitters of type $r \in \mathcal{C}^{r}$, installed/extracted from the central bundle node $N^{B C}$. Continuing, we apply the same rule introducing pairs $\left(y_{i n s}^{C j}, y_{\text {ext }}^{C j}\right)$ and $\left(z_{\text {ins }}^{C j}, z_{\text {ext }}^{C j}\right)$ to denote variables counting, respectively, OLT cards and OLT devices that need to be installedlextracted at transition $j$. Finally, we introduce variables $b^{a j} \in\{0,1\}, b^{d j} \in\{0,1\}$, and $b^{C j} \in\{0,1\}$ to indicate if a particular (either access $a \in \mathcal{N}^{B A}$, distribution $d \in \mathcal{N}^{B D}$ or central $n^{B C}$ ) bundle node, requires site-survey at transition $j \in \mathcal{J}$. The site survey is necessary if any change of the nodal equipment is required in it (either installation or extraction). Having all this defined, we introduce the following vectors:

$\mathbf{x}_{i n s}^{A j}=\left(x_{i n s}^{a j w}: a \in \mathcal{N}^{B A}, w \in \mathcal{C}^{r}\right)$,

$\mathbf{x}_{\text {ext }}^{A j}=\left(x_{\text {ext }}^{a j w}: a \in \mathcal{N}^{B A}, w \in \mathcal{C}^{r}\right)$,

$\mathbf{x}_{\text {ins }}^{D j}=\left(x_{\text {ins }}^{d j s}: d \in \mathcal{N}^{B D}, s \in \mathcal{C}^{r}\right)$,

$\mathbf{x}_{\text {ext }}^{D j}=\left(x_{\text {ext }}^{d j s}: d \in \mathcal{N}^{B D}, s \in \mathcal{C}^{r}\right)$,

$\mathbf{x}_{\text {ins }}^{C j}=\left(x_{i n s}^{C j r}: r \in \mathcal{C}^{r}\right)$,

$\mathbf{x}_{\text {ext }}^{C j}=\left(x_{\text {ext }}^{C j r}: r \in \mathcal{C}^{r}\right)$,

$\mathbf{b}^{A j}=\left(b^{a j}: a \in \mathcal{N}^{B A}\right)$,

$\mathbf{b}^{D j}=\left(b^{d j}: d \in N^{B D}\right)$,

$\mathbf{b}^{C j}=\left(b^{C j}\right)$.

For any period transition $j \in \mathcal{J}$, we refer to vector (7) as the reconfiguration between configurations $X^{k=a(j)}$ to $X^{t=z(j)}$.

$$
\begin{aligned}
\mathbf{H}^{j}= & \left(\mathbf{x}_{\text {ins }}^{A j}, \mathbf{x}_{\text {ext }}^{A j}, \mathbf{x}_{\text {ins }}^{D j}, \mathbf{x}_{\text {ext }}^{D j}, \mathbf{x}_{\text {ins }}^{C j}, \mathbf{x}_{\text {ext }}^{C j},\right. \\
& \left.\mathbf{y}_{\text {ins }}^{C j}, \mathbf{y}_{\text {ext }}^{C j}, \mathbf{z}_{\text {ins }}^{C j}, \mathbf{z}_{\text {ext }}^{C j}, \mathbf{b}^{A j}, \mathbf{b}^{D j}, \mathbf{b}^{C j}\right) .
\end{aligned}
$$

Then, we define set $\mathscr{H}^{j}$-we refer to it as inter-period feasible set at transition $j$-containing every feasible reconfiguration between these configurations (detailed description of the set is presented in Sect. 5.4).

Now we can formulate the multi-period optimization problem in the following way:

$$
\begin{aligned}
\min F= & \sum_{t \in \mathcal{T}} C^{E-N}\left(\mathbf{X}^{\mathbf{t}}\right)+\sum_{t \in \mathcal{T}} C^{I-N}\left(\mathbf{X}^{t}\right) \\
& +\sum_{j \in \mathcal{J}} C^{N P}\left(\mathbf{H}^{j}\right)
\end{aligned}
$$




$$
\begin{array}{ll}
\text { s.t. } & \\
\mathbf{X}^{t} \in \mathscr{X}^{t}, & t \in \mathcal{T} \backslash\{0\}, \\
\mathbf{X}^{0}=\mathbf{0}, & \\
\mathbf{H}^{j} \in \mathscr{H}^{j}, & j \in \mathcal{J} .
\end{array}
$$

Observe that optimization problem (8) has a special structureone can distinguish the intra-period specific part (we refer to it as intra-period problem) defined by constraints ( $8 \mathrm{c}$ and $8 \mathrm{~d}$ ), and the inter-period specific part (inter-period problem) constituted by constraint (8e). The intra-period part has the associated objective function components representing the $E-N$ (see Sect. 4.4.1) and $I-N$ (see Sect. 4.4.2) elements of the NP role's cost (8a). The inter-period part has only the objective component ( $8 \mathrm{~b}$ ) associated with the NP cost component (see Sect. 4.4.3). Constraints (8d) are just to indicate that the green-field deployment is considered, as the initial configuration $X^{0}$ has no equipment installed. Although we do not exploit this special structure to reformulate the problem, we use it to structuralize the problem description-feasible intra-period polyhedron and the associated components of the objective function are described in Sects. 5.2 and 5.3; the same information related to the inter-period part can be found in Sects. 5.4 and 5.5

\subsection{Intra-period problem: feasible set}

The feasible polyhedron $\mathscr{X}^{t}$ (see Sect. 5.1) of a single time period $t \in \mathcal{T} \backslash\{0\}$, is described by means of a number of constraints. To facilitate reading, we split these constraints into groups related to particular levels of the network hierarchycentral, distribution, and access. Taking into account the amount of variables and parameters necessary to formulate the problem we decided to introduce them in places when they are used (each time we refer a variable introduced in the preceding sections, we try to put a related cross-reference to its definition).

Formulating the intra-period problem, we assume the following:

- there is exactly one central bundle node $n^{B C}$,

- access bundle node $a \in \mathcal{N}^{B A}$, is connected to exactly one distribution bundle node $b \in \mathcal{N}^{B D}$; the assignment is given as an input,

- all signal network connections that satisfy demand of a given access bundle node $b \in \mathcal{N}^{B A}$, share the same infrastructure trails,

- the physical length of every infrastructure trail is given; thus, the distance from each access bundle node $b \in$ $\mathcal{N}^{B A}$, to the head-end bundle node is also known,

- only symmetrical splitters with uniform splitting ratios are admissible.
We start the description of constraints from the central node $n^{B C}$ and continue downward the network hierarchy through distribution nodes $d \in \mathcal{N}^{B D}$, down to access bundle nodes $a \in \mathcal{N}^{B A}$.

\subsubsection{Central site constraints}

For the central bundle node $n^{B C}$ and each time period $t \in$ $\mathcal{T} \backslash\{0\}$, the following constraints must hold:

$$
\begin{array}{rlrl}
\sum_{d \in \mathcal{N}^{B D}} k^{d t r} & \leq x^{C t r} \eta^{r}, & r \in \mathcal{C}^{r}, \\
\sum_{d \in \mathcal{N}^{B D}} \hat{k}^{d t r} & \leq \hat{x}^{C t r} \eta^{r}, \quad r \in \mathcal{C}^{r}, \\
\sum_{r \in \mathcal{C}^{r}} x^{C t r} & \leq y^{C t} \eta^{C}, & \\
y^{C t} & \leq z^{C t} \eta^{O} .
\end{array}
$$

Variables $k^{d t r} \in \mathbb{R}_{+}$, and $\hat{k}^{d t r} \in \mathbb{R}_{+}$, represent the number of real/virtual trunk fibre paths leading from the central node to every distribution node $d \in \mathcal{N}^{B D}$ (see Sect. 3) connected in $n^{B C}$ to ports of real/virtual splitters of type $r \in \mathcal{C}^{r}$, that are necessary to satisfy requirements (demand) of each distribution bundle node $d \in \mathcal{N}^{B C}$. Constraints (9a) and (9b) enforce installation of real/virtual splitters in the number that is sufficient w.r.t. a demand of every distribution node. Constraint (9c) guarantees that there is sufficient number of OLT cards to connect every real splitter (virtual splitters of $n^{B C}$ are not considered as, by definition, they remain not connected - see Sect. 5.1). Finally, constraint (9d) enforces that the capacity of installed OLT devices is sufficient to accommodate the required number of OLT cards.

\subsubsection{Distribution site constraints}

For each pair $(d, t) \in \mathcal{N}^{B D} \times \mathcal{T} \backslash\{0\}$, distribution bundle node $d$ and time period $t$, the following constraints must hold (notion $c(d), d \in \mathcal{N}^{B d}$, denotes here a set of access bundle nodes connected to distribution bundle node $d$ ):

$$
\begin{array}{rlrl}
\sum_{a \in c(d)} k^{d a t r s} \leq l^{d t r s} \eta^{s}, & (r, s) \in \mathcal{C}^{2 r}, \\
\sum_{a \in c(d)} \hat{k}^{d a t r s} \leq \hat{l}^{d t r s} \eta^{s}, & (r, s) \in \mathcal{C}^{2 r}, \\
\sum_{r:(r, s) \in C^{2 r}} l \leq x^{d t s}, & s \in \mathcal{C}^{r}, \\
\sum_{r:(r, s) \in C^{2 r}} l^{d t r s}+\hat{l}^{d t r s} & =\left(x^{d t s}+\hat{x}^{d t s}\right), s \in \mathcal{C}^{r}, \\
\sum_{s:(r, s) \in \mathcal{C}^{2 r}} l^{d t r s} & \leq k^{d t r}, & r \in \mathcal{C}^{r},
\end{array}
$$




$$
\sum_{s:(r, s) \in C^{2 r}} l^{d t r s}+\hat{l}^{d t r s} \leq\left(k^{d t r}+\hat{k}^{d t r}\right), r \in \mathcal{C}^{r} .
$$

Variables $k^{\text {datrs }} \in \mathbb{R}_{+}$, and $\hat{k}^{\text {datrs }} \in \mathbb{R}_{+}$, represent the number of real/virtual distribution fibre paths (between access node $a \in \mathcal{N}^{B A}$, and its superior distribution node $d \in \mathcal{N}^{B D}: a \in$ $c(d))$ that in the central and distribution nodes are served by splitters of respective types $r \in \mathcal{C}^{r}$, and $s \in \mathcal{C}^{r},(r, s) \in \mathcal{C}^{2 r}$. Observe that these variables, which are as numerous as access points of the considered network, are kept rational, not integral. The maneuver facilitates resolution of the resulting MIP problem. To preserve eventual integrity of the solution, we introduce auxiliary-integer, still one order of magnitudeless numerous-variables $l^{d t r s} \in \mathbb{Z}_{+}$, and $\hat{l}^{\text {dtrs }} \in \mathbb{Z}_{+}$, $d \in \mathcal{N}^{B D}, \quad t \in \mathcal{T}, \quad(r, s) \in \mathcal{C}^{2 r}$, which are to count the number of trunk fibre paths being connected to a pair $(r, s) \in \mathcal{C}^{2 r}$, of splitters in the central and distribution bundle nodes, respectively. Consistency between these two pairs of variables, both in the real and the virtual case, is preserved by constraints (10a) and (10b). Constraints (10c) and (10d) guarantee that the sufficient numbers of real/virtual splitters of each type $s \in \mathcal{C}^{r}$, are installed in the distribution node (remind that real distribution splitters must be connected to real central splitters, while virtual distribution splitters can be connected to either real or virtual central splitters). Finally, constraints (10e) and (10f) are to enforce proper behavior of $k^{d t r}$ and $\hat{k}$ variables that, as we remember from Sect. 5.2.1, drive the central node to install the sufficient number of OLT cards and OLT devices.

To facilitate describing the objective function, we introduce two auxiliary variables $-x^{d t} \in \mathbb{R}_{+}, d \in \mathcal{N}^{B D}$, and $\hat{x}^{d t} \in \mathbb{R}_{+}, d \in \mathcal{N}^{B D}$, that are to count the total number of real/virtual splitters installed in the distribution node. These variables are set to proper values thanks to the following constraints:

$$
\begin{aligned}
& x^{d t}=\sum_{s \in \mathcal{C}^{r}} x^{d t s}, \\
& \hat{x}^{d t}=\sum_{s \in \mathcal{C}^{r}} \hat{x}^{d t s} .
\end{aligned}
$$

\subsubsection{Access constraints}

For each pair of an access node and a time period $(a, t) \in$ $\mathcal{N}^{B A} \times \mathcal{T} \backslash\{0\}$, the following constraints must hold:

$$
\begin{aligned}
h^{B t a} & \leq \sum_{w \in \mathcal{C}^{r}} x^{a t w} \eta^{w}, \\
x^{a t} & =\sum_{w \in \mathcal{C}^{r}} x^{a t w}, \\
\hat{x}^{a t} & =\sum_{w \in \mathcal{C}^{r}} \hat{x}^{a t w},
\end{aligned}
$$

$$
\begin{aligned}
x^{a t} & \leq \sum_{(r, s) \in \mathcal{C}^{2 r}} k^{\text {datrs }}, \quad d: a \in c(d), \\
x^{a t}+\hat{x}^{a t} & \leq \sum_{(r, s) \in \mathcal{C}^{2 r}}\left(k^{\text {datrs }}+\hat{k}^{\text {datrs }}\right), d: a \in c(d), \\
x^{a t w} & \leq \sum_{(r, s, w) \in \mathcal{C}^{3 r}} k^{\text {datrs }}, d: a \in c(d), w \in \mathcal{C}^{r} .
\end{aligned}
$$

Constraint (12a) guarantees that in every access node $n \in$ $\mathcal{N}^{B A}$, in every time period $t \in \mathcal{T} \backslash\{0\}$, the number of signal ports provided by real splitters satisfies the demand for optical signals $h^{B t a}$ (see Sect. 3.4). To facilitate reading, constraints (12b) and (12c) introduce variables $x^{a t} \in \mathbb{R}_{+}$, and $\hat{x}^{a t} \in \mathbb{R}_{+}$, to count the total number of real/virtual splitters and enforce consistency of their values. Then constraints (12d) and (12e) impose that the number of real splitters and the total number of splitters (both real and virtual) are lower than, respectively, the number of real distribution fibre paths and the total number of distribution paths (again, real and virtual). Finally, constraint (12f) imposes that the number of distribution fibre paths, which according to the set of admissible splitter triples $\mathcal{C}^{3 r}$ can serve every splitter of type $w \in \mathcal{C}^{r}$, is large enough to serve the installed splitters of this type.

\subsection{Intra-period problem: state cost}

The intra-period cost, see (8b) in formulation (8), depends solely on the single state $\mathbf{X}^{t}$ and represents the cost of leasing resources at $E-N$ and $I-N$ interfaces (see Sect. 4.2) in this period. It is expressed by the following equations:

$$
\begin{aligned}
C^{E-N}\left(\mathbf{X}^{t}\right)= & \\
& +y^{C t} \zeta^{C}+z^{C t} \zeta^{O} \\
& +\sum_{r \in \mathcal{C}^{r}} x^{C t r} \zeta^{r} \\
& +\sum_{d \in \mathcal{N}^{B D}} \sum_{s \in \mathcal{C}^{r}} x^{d t s} \zeta^{s} \\
& +\sum_{a \in \mathcal{N}^{B A}} \sum_{w \in \mathcal{C}^{r}} x^{a t w} \zeta^{w}
\end{aligned}
$$

We split the component into two parts related, respectively, to $E-N$ and $I-N$ business interfaces. First, at $E-N$ interface, between the network provider and the equipment provider role (see Sect. 4.4), we consider costs of OLT devices and OLT cards (13a) as well as costs of optical splitters installed in the central (13b), distribution (13c), and access (13d) nodes.

$$
\begin{aligned}
C^{I-N}\left(\mathbf{X}^{t}\right)= & \\
& +\sum_{r \in \mathcal{C}^{r}} x^{C t r}\left(\eta^{r}+1\right) \zeta_{C}^{R} \\
& +\sum_{r \in \mathcal{C}^{r}} x^{C t r} \zeta_{\text {port }}^{O}
\end{aligned}
$$




$$
\begin{aligned}
& +\sum_{d \in \mathcal{N}^{B D}} \sum_{s \in \mathcal{C}^{r}} x^{d t s}\left(\eta^{s}+1\right) \zeta_{d}^{R} \\
& +\sum_{d \in \mathcal{N}^{B D}} \sum_{s \in \mathcal{C}^{r}} x^{d t s} \zeta_{d}^{F T} \\
& +\sum_{a \in \mathcal{N}^{B A}} \sum_{w \in \mathcal{C}^{r}} x^{a t w}\left(\eta^{w}+1\right) \zeta_{a}^{R} \\
& +\sum_{a \in \mathcal{N}^{B A}} \sum_{w \in \mathcal{C}^{r}} x^{a t w} \zeta_{a}^{F D} .
\end{aligned}
$$

Second, at $I-N$ interface, between the network provider and the passive infrastructure provider role, we cost the exploitation (by installed splitters) of cabinet's space at the central (14a), distribution (14c), and access nodes (14e), as well as exploitation of PIP's communication infrastructureinstallation of OLT port (14b) and access to a trunk (14d) and distribution (14f) fibre paths.

\subsection{Inter-period problem: feasible set}

This section is to describe polyhedrons $\mathscr{H}^{j}, j \in \mathcal{J}$, (see Sect. 5.1) that comprise admissible values for vectors $\mathbf{H}^{j}$. We emphasis that we consider a green-field design-we identified the initial period $t=0$ with no equipment installed in any node of the network-see constraint (8d). Similarly to the intra-period problem, constraints are grouped according to the level of the considered network they pertain to.

\subsubsection{Central site constraints}

For central bundle node $n^{B C}$ and each pair of consecutive time periods $j=(k, t)$ of transition $j \in \mathcal{J}$, the following constraints must hold:

$$
\begin{aligned}
x_{\text {ins }}^{C j r} & \geq x^{C t r}+\hat{x}^{C t r} & \\
& -\left(x^{C k r}+\hat{x}^{C k r}\right), & r \in \mathcal{C}^{r}, \\
x_{\text {ext }}^{C j r} \geq x^{C k r}+\hat{x}^{C k r} & & \\
y^{C t} & \left.=y_{\text {ins }}^{C j}-x_{\text {ext }}^{C t r}+\hat{x}^{C t r}\right), & r \in \mathcal{C}^{r}, \\
z^{C t} & =z_{\text {ins }}^{C j}-z_{\text {ext }}^{C j}+z^{C k}, & \\
M b^{C j} & \geq y_{\text {ins }}^{C j}+y_{\text {ext }}^{C j}+z_{\text {ins }}^{C j}+z_{\text {ext }}^{C j} & \\
& +\sum_{r \in \mathcal{C}^{r}}\left(x_{\text {ins }}^{C j r}+x_{\text {ext }}^{C j r}\right) . &
\end{aligned}
$$

Constraints (15a) and (15b) enforce correct behavior of variables $x_{i n s}^{C j r}$ and $x_{\text {ext }}^{C j r}$ counting, respectively, the number of installed and extracted splitters of each type $r \in \mathcal{C}^{r}$, (observe that both real and virtual splitters are counted together). Constraints (15c) and (15d) impose consistency between numbers of OLT devices and OLT cards (represented by variables $y^{C t}$ and $z^{C t}$ ) and the number of OLT devices and OLT cards installed and extracted in every time period. Finally, constraint (15e) sets the site-survey flag for the central node (the big- $M$ constant used in-there is not explicitly exposed in the problem sent to the solver-we use the notion of an indicator constraint instead).

\subsubsection{Distribution constraints}

For each distribution bundle node $d \in \mathcal{N}^{B D}$, and each pair of consecutive time periods $j=(k, t)$ of transition $j \in \mathcal{J}$, the following constraints must hold:

$$
\begin{array}{rlr}
x_{i n s}^{d j s} \geq & x^{d t s}+\hat{x}^{d t s} \\
& -\left(x^{d k s}+\hat{x}^{d k s}\right), & \\
x_{\text {ext }}^{d j s} \geq & x^{d k s}+\hat{x}^{d k s} & \\
& -\left(x^{d t s}+\hat{x}^{d t s}\right), & \\
M b^{j t} & \geq \sum_{s \in \mathcal{C}^{r}}\left(x_{\text {ins }}^{d j s}+x_{\text {ext }}^{d j s}\right) . &
\end{array}
$$

Constraints (16a) and (16b) enforce correct behavior of variables $x_{i n s}^{d j s}$ and $x_{\text {ext }}^{d j s}$ counting, respectively, the number of installed and extracted splitters of each type $s \in \mathcal{C}^{r}$, (observe that both real and virtual splitters are counted together). Finally, constraint (16c) sets the site-survey flag for the distribution node (as in the case of the central node, in the actual problem sent to the solver, the indicator constraint is used instead of the big- $M$ constant).

\subsubsection{Access constraints}

For each access bundle node $a \in \mathcal{N}^{B A}$, each pair of consecutive time periods $j=(k, t)$ of transition $j \in \mathcal{J}$, the following constraints must hold:

$$
\begin{aligned}
x_{\text {ins }}^{a j w} \geq & x^{a t w}+\hat{x}^{a t w} & \\
& -\left(x^{a k w}+\hat{x}^{a k w}\right), & \\
x_{\text {ext }}^{a j w} \geq & x^{a k w}+\hat{x}^{a k w} & \\
M b^{a j} & \geq \sum_{w \in \mathcal{C}^{r}}\left(x^{a t w}+\hat{x}^{a t w}\right), &
\end{aligned}
$$

Constraints (17a) and (17b) enforce correct behavior of variables $x_{i n s}^{a j w}$ and $x_{\text {ext }}^{a j w}$ counting, respectively, the number of installed and extracted splitters of each type $w \in \mathcal{C}^{r}$, (observe that both real and virtual splitters are counted together). Finally, constraint (17c) sets the site-survey flag for the access node (as in the case of the central and distribution nodes, in the actual problem sent to the solver, the indicator constraint is used instead of the big- $M$ constant). 


\subsection{Inter-period problem: transition cost}

To facilitate description of the transition cost (8b) we introduce the following symbols related to the particular cost components. We emphasise that all these components are discrete in opposition to lease cost identified in the intraperiod problem.

$-c_{i n t}^{C}$ - cost of site-survey in central site $n^{B C}$,

$-c_{i n t}^{D}$-common cost of site-survey in every distribution site $d \in \mathcal{N}^{I S D}$,

$-c_{i n t}^{A}$-common cost of site-survey in every access site $a \in \mathcal{N}^{I S A}$,

$-c_{i n s}^{O}, c_{\text {ext }}^{O}$-cost of OLT device installation/extraction,

$-c_{i n s}^{C}, c_{e x t}^{C}$ - cost of OLT-card installation/extraction,

$-c_{i n s}^{r}, c_{e x t}^{r}, r \in \mathcal{C}^{r}$ - cost of installation/extraction of splitter of type $r \in \mathcal{C}^{r}$ in every node.

Having the symbols defined, we can finally write the related cost expression for every transition $j \in \mathcal{J}$, as follows:

$$
\begin{aligned}
C^{N P}\left(\mathbf{H}^{j}\right)= & \\
& +y_{i n s}^{C j} c_{\text {ins }}^{C}+y_{\text {ext }}^{C j} c_{\text {ext }}^{C} \\
& +z_{\text {ins }}^{C j} c_{\text {ins }}^{O}+z_{\text {ext }}^{C j} c_{\text {ext }}^{O} \\
& +\sum_{r \in \mathcal{C}^{r}}\left(x_{i n s}^{C j r} c_{\text {ins }}^{r}+x_{\text {ext }}^{C j r} c_{\text {ext }}^{r}\right) \\
& +b^{C j} c_{i n t}^{C} \\
& +\sum_{d \in \mathcal{N}^{B D}} \sum_{s \in \mathcal{C}^{r}}\left(x_{i n s}^{d j s} c_{i n s}^{s}+x_{\text {ext }}^{d j s} c_{\text {ext }}^{s}\right) \\
& +\sum_{d \in \mathcal{N}^{B D}} b^{d j} c_{i n t}^{D} \\
& +\sum_{a \in \mathcal{N}^{B A}} \sum_{w \in \mathcal{C}^{r}}\left(x_{i n s}^{a j w} c_{i n s}^{w}+x_{\text {ext }}^{a j w} c_{\text {ext }}^{w}\right) \\
& +\sum_{a \in \mathcal{N}^{B A}} b^{a j} c_{i n t}^{A} .
\end{aligned}
$$

Expressions (18a) and (18b) represent costs of installation/extraction of OLT devices and OLT cards. Remind that in our research we assume only one type of devices and cards. Expressions (18c), (18e), and (18g) represent cost components related to installation/extraction of optical splitters of particular types in, respectively, central, distribution, and access bundle nodes. Finally, expressions (18d), (18f), and $(18 \mathrm{~h})$ indicate costs of site-surveys for the central, distribution, and access bundle nodes.

\section{Numerical experiments}

In this section, we report results of our numerical experiments aimed at proving the validity of our approach and the correctness of our implementation. The section comprises three parts-first, Sect. 6.1 describes how values of particular parameters were estimated; second, in Sect. 6.2, we indicate the way we managed the large sets of variables and constraints of the problem (see Sect. 5); and third, in Sect. 6.3, we present and conclude results of the conducted experiments.

\subsection{Setting parameter values}

The formulation presented in Sect. 5 takes advantage on numerous parameters related to the cost of particular resources as well as to the customer demand (i.e., the time, location, and the number of optical signals the network is expected to provide). As these parameters can have the crucial impact on the solution, their values must be set with the utmost consideration.

In the reported experiments, we consider a single trajectory of the customer arrival process. Information on the final (observed in the sixteenth period) values of optical signal demand in each access point we took from our previous research devoted to one-step network deployment, see [23], while the arrival times are modeled using a logistic function, which is commonly used to model diffusion of innovations [27]. The aggregated result of the estimation, i.e, the total number of optical signal the network is expected to deliver in particular time periods, is presented in Fig. 10.

The cost parameters define charges for one-period exploitation of product resources exposed at the $E N$ and $H N$ business interfaces (see Sect. 4) as well as OPEX expenditures carried out by the NP role on the period-by-period basis. To estimate one-period charges for the product resources offered at the $E-N$ business interface, i.e., OLT devices, OLT cards, and optical splitters, we take advantage of price information from the equipment catalog (see Sect. 3.6). Under the assumption that the considered set of periods $\mathcal{T}$ covers the whole life-

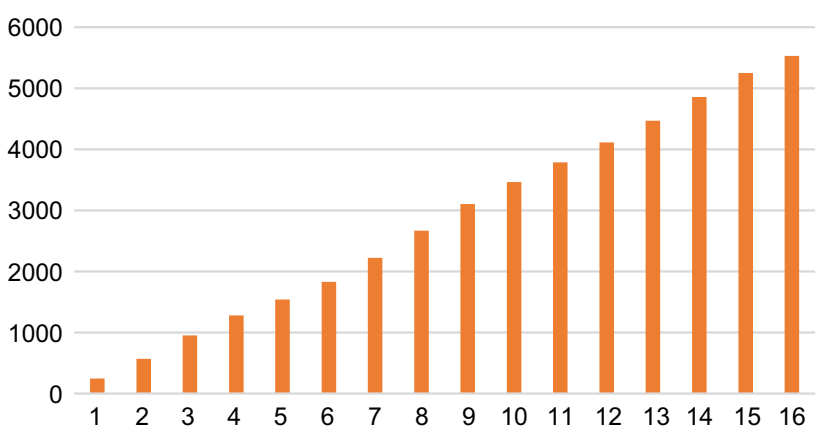

Fig. 10 Required number of optical signals (total demand) 


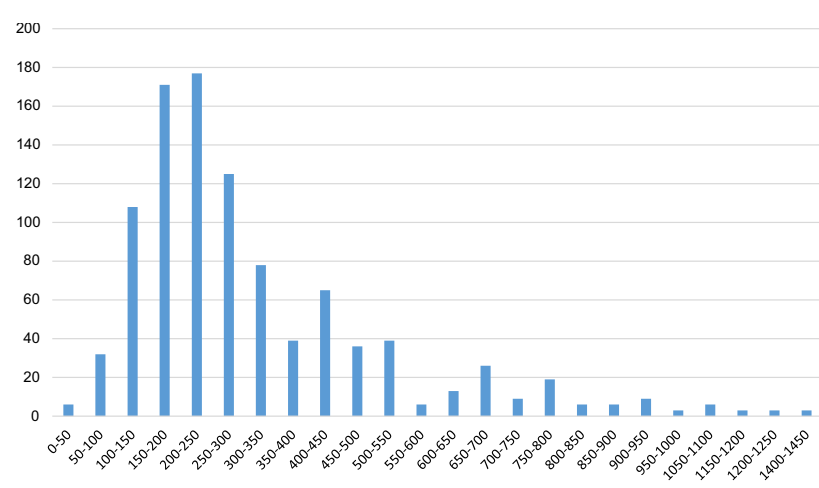

Fig. 11 Distribution of distribution fibre-path single-period charge

time of passive resources (and, its is twice as long as the expected lifetime active ones), applying the linear depreciation model (see Sect. 4.3), zero salvage value, and neglecting any other (like insurance, etc.) cost, we obtain an estimation of one-period resource charge dividing the catalog price (the procurement cost) of the resource by the number of timeperiods it is expected to last.

Estimation of one-period charges for product resources offered at the $I-N$ interface-i.e., trunk and distribution fibre paths; cabinet space in central, distribution, and access sites; allocation of a individual OLT ports-is more complicated due to the fact that these resources are build on the top of shared supporting infrastructure, and there is no one-to-one relation between each of them and any individual network element. To cope with this issue, we apply a two-step approach-first, we estimate reasonable deployment costs of individual network elements; second, we project these costs onto product resources they support. To complete the first step, we solve, with advantage of methods and tools elaborated in our previous research [23], an auxiliary problem that aims at minimizing the total cost of one-step network deployment. To estimate one-period charge for trunk and distribution fibre paths, we take advantage of the assumption that there is defined at most one infrastructure trail between a pair of sites; the trail is then used for realization of every fibre paths between bundle nodes located in these sites. Consider an infrastructure link, e.g., duct or overhead line, $l \in \mathcal{L}^{I}$. A solution of the auxiliary problem gives us the cost of preparation of this link, the number and types of optical cables that are hosted in-there, and the number of active fibres they carry. With this information, we can estimate a fraction of the link cost associated with a single active fibre. Consider a set of distribution fibre paths that enter bundle node $a \in \mathcal{N}^{B A}$. Every of these paths uses the same way to the same distribution bundle node, hence every path accumulates a per-activefibre cost of every traversed infrastructure link. Finally, we receive the one-period charge dividing the result cost by the number of time periods in the considered network lifetime. Figure 11 presents a distribution of distribution fibre one-

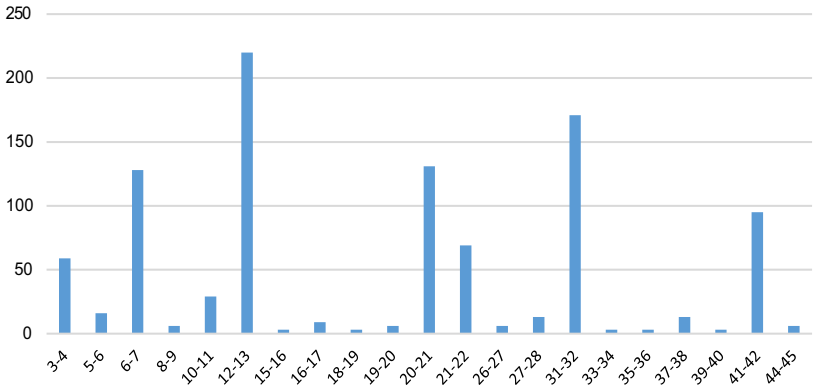

Fig. 12 Distribution of distribution site port single-period charge

period charges; the $\mathrm{x}$-axis is denominated in cost ranges, while the $y$-axis shows a fraction of bundle nodes that are exposed to fibre costs fitting to particular ranges (expressed in parts per thousand). The one-period charges for trunk fibre paths can be estimated in a similar way.

The one-period charge for space of sites, which host central, distribution, and access bundle node, that can be used to accommodate optical splitters is estimated in a similar way. For each site $n \in \mathcal{N}^{I S}$, the solution of the auxiliary optimization problem identifies its type $f \in \mathcal{C}^{f}$, (building, in-building cabinet, street-cabinet, etc.), preparation $\operatorname{cost} c^{f}$ as well as cost $c^{e}$ and capacity $\eta^{e}$ of every hardware rack (cabinet) of type $e \in \mathcal{C}^{e}$, installed in-there. In our costprojection scheme, we simply divide the total site-related cost by the number of ports of splitters installed in its hosted cabinets (and by the number of time periods in the considered network lifetime) consequently receiving an estimation of the single-period per-port charge (distribution of the singleperiod distribution port charge, received for the considered case, is presented in Fig. 12).

The only site where active equipment (OLT and OLT cards) is installed is the central site $n^{I S C}$; presence of the OLT device—requiring power supplying, cooling, securitygenerates additional OLT-specific costs that the PIP role has to project onto product resources. To cope with this cost, we introduce a product referred to as OLT port access that expresses per-period cost of the OLT site preparation; it is computed by dividing a cost of the central site preparation by the number of active ports installed in the OLT device according to the optimal solution of the auxiliary problem.

It has to be emphasized that the single-period optimization problem takes at its input the same price-list as used for costing products at the $E-N$ business interface (equipment and labor costs were taken from [1]) and thus, prices at the $E-N$ and $I-N$ interfaces constitute a consistent costing system.

Estimation of transition-related costs is more complicated-its every component, i.e., site-survey cost and equipment installation/extraction cost has to be set in consistency with $E-N / I-N$ system-as otherwise one of the configuration-related and transition-related components 
might overweight results. Provided the price-lists expose the real-world costs, it is relatively easy to estimate reasonable cost of site-surveys (this is the way we did it for our experiments). Estimation of the cost of equipment installation/extraction, as it reflects both cost of the related manual intervention as well as complexity of operation to be done, is vaguer. For our experiment, we set these parameters arbitrarily, with the assumption that installation/extraction incurs constant (proportional to the catalogue price of the involved equipment) and varying (proportional to the number of external ports of the equipment) cost components.

\subsection{Maneuvers and tricks}

In our implementation, we exploit AMPL modeling language [15] with the CPLEX solver. Both input data and the optimal solution are kept in Microsoft SQL database; catalog data is stored in the Microsoft Excel files. The general scenario of the computations is presented in Algorithm 1. First (steps 1 and 2), data describing physical and logical organization of the network is read-in from the database and Excel files. In step 3, we solve the auxiliary one-step deployment problem that gives us an optimal solution and allows for estimation of the cost parameter values (step 4). Then, in step 5, we read (from the database) the trajectory of the customer arrival process that describes number of optical signals the network is required to provide in every particular access point in every time period (preparation of these data is briefly described in Sect. 6.1).

As considered problem instances were too large (in step 15 of the algorithm, the problem passed to Cplex contains about 240000 variables-4864 binary and 68802 integer-and 135000 constraints) to be approached directly — doing in that way we were not able to receive even root-node relaxationswe adopted the following solution. First, in steps $6-9$, we solve, one-by-one, a set of auxiliary single-period problems. A solution of each auxiliary problem $\mathcal{P}^{t}, t \in \mathcal{T}$, is then used to fix the $t$-period-related variables of the original problem $\mathcal{P}$. Taking advantage of the assumption that demand of each particular access point does not fall in consecutive time periods, to speed up the computations, we solve the auxiliary problems (in the reverse order of elements of set $\mathcal{T}$ ) starting from the last one with the highest demand. Consequently, an optimal solution of problem $\mathcal{P}^{t}$ constitutes a feasible solution of problem $\mathcal{P}^{t-1}$. This fact can be exploited by the used MIP solver in the warm-start procedure. In step 10 , we receive a feasible solution of the complete problem $\mathcal{P}$ with variables fixed according to the solutions of the auxiliary problems; these restrictions are gradually (period by period) removed in steps 11-14 (we observed that performing in this way, we receive faster progress than in the case all the restrictions are removed simultaneously). In step 15, we solve whole problem $\mathcal{P}$ with all the previously introduced restrictions removed until optimality gap of $5 \%$ has been reached (thanks to the described tricks, Cplex is able to perform the warm-start with known integer solution). Finally, the received solution is written into the database and then undergoes the final processing.

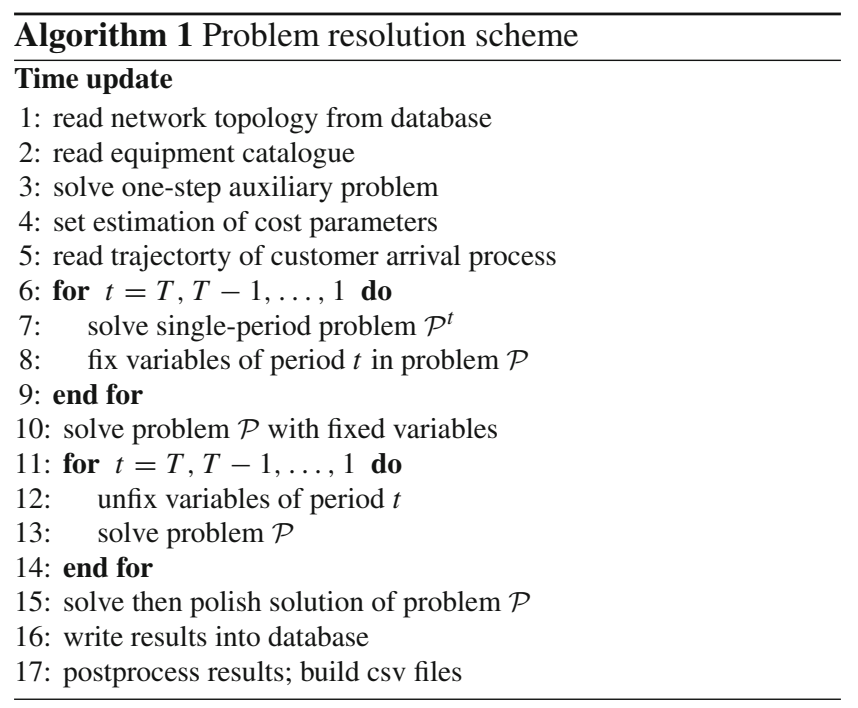

\subsection{Results of experiments}

In our preliminary experiments, we considered one network topology consisting of a single central bundle node, two distribution bundle nodes, and 304 access bundle nodes; the lifespan has been divided into sixteen time periods of equal length. All the reported experiments were conducted on Hewlett-Packard HP DL380 G9 server (Xeon 10C processors) using AMPL and CPLEX 12.7 accessing up-to eight logical processors and up-to 64 GB RAM. We present results for three selected experiments-referred hereafter to as: $C C$ experiment, CTC experiment, and CC-PIR experiment - that shared the same customer arrival trajectory differing in terms of objective functions applied and/or presence of transitionrelated constraints. Every experiment was terminated when a 5\% gap has been reached.

The CC experiment aims at minimizing solely configuration costs (see Sect. 5.3) and permitting for arbitrarily large reconfigurations between consecutive periods (see the stub release approach in Sect. 1). This experiment is expected to identify the lower bound for the sum of the configurationrelated costs. Next, the CTC experiment minimizes every cost component, i.e., configuration (see Sect. 5.3) as well as reconfiguration related (see Sect. 5.5), providing a reference optimal solution that can be used to evaluate the quality of results provided by simpler, thus computationally more effective, approaches. Finally, the CC-PIR experiment, similarly to the $\mathrm{CC}$ experiment, considers configuration-related costs, 
Table 4 Configuration cost optimization [CC part A]—raw result costs (optimality gap 0.05)

\begin{tabular}{rrrllllllllrrrrrrr}
\hline $\mathrm{t}$ & $\mathrm{h}$ & $\mathrm{hi}$ & $\mathrm{hvi}$ & $\mathrm{cs}$ & $\mathrm{csv}$ & $\mathrm{csI}$ & $\mathrm{csE}$ & $\mathrm{ds}$ & $\mathrm{dsv}$ & $\mathrm{dsI}$ & $\mathrm{dsE}$ & as & asv & asI & asE & o \\
\hline 1 & 247 & 303 & 0 & 5 & 3 & 280 & 0 & 187 & 0 & 1920 & 0 & 448 & 0 & 5170 & 0 & 6750 \\
2 & 571 & 684 & 0 & 11 & 4 & 245 & 0 & 182 & 0 & 640 & 1325 & 897 & 0 & 7530 & 5265 & 6750 \\
3 & 952 & 1070 & 0 & 17 & 5 & 245 & 0 & 340 & 0 & 1625 & 0 & 1345 & 0 & 9550 & 8560 & 6750 \\
4 & 1279 & 1460 & 0 & 23 & 6 & 245 & 0 & 315 & 0 & 540 & 1325 & 1771 & 0 & 10445 & 10385 & 6750 \\
5 & 1542 & 1763 & 0 & 28 & 4 & 105 & 0 & 319 & 0 & 280 & 365 & 2133 & 0 & 7940 & 6650 & 6750 \\
6 & 1827 & 2045 & 0 & 32 & 6 & 210 & 0 & 435 & 0 & 1150 & 0 & 2522 & 0 & 9680 & 9735 & 6750 \\
7 & 2220 & 2526 & 0 & 40 & 3 & 175 & 0 & 397 & 0 & 350 & 1325 & 3070 & 0 & 11075 & 9290 & 13500 \\
8 & 2666 & 3046 & 0 & 48 & 3 & 280 & 0 & 434 & 0 & 420 & 0 & 3646 & 0 & 12520 & 11325 & 13500 \\
9 & 3102 & 3558 & 0 & 56 & 4 & 315 & 0 & 542 & 0 & 1175 & 0 & 4274 & 0 & 13050 & 11395 & 13500 \\
10 & 3464 & 3817 & 0 & 60 & 4 & 140 & 0 & 595 & 0 & 500 & 0 & 4677 & 0 & 12250 & 14220 & 13500 \\
11 & 3788 & 4160 & 0 & 65 & 4 & 175 & 0 & 644 & 0 & 530 & 85 & 5179 & 0 & 9945 & 8750 & 20250 \\
12 & 4113 & 4605 & 0 & 72 & 4 & 245 & 0 & 686 & 0 & 580 & 365 & 5694 & 0 & 12460 & 12475 & 20250 \\
13 & 4466 & 4943 & 0 & 78 & 4 & 210 & 0 & 767 & 0 & 850 & 0 & 6140 & 0 & 11375 & 11830 & 20250 \\
14 & 4855 & 5376 & 0 & 84 & 5 & 245 & 0 & 803 & 0 & 405 & 0 & 6652 & 0 & 10350 & 9135 & 20250 \\
15 & 5249 & 5798 & 0 & 91 & 6 & 280 & 0 & 864 & 0 & 600 & 0 & 7148 & 0 & 10420 & 9840 & 20250 \\
16 & 5531 & 6099 & 0 & 96 & 4 & 105 & 0 & 837 & 0 & 215 & 810 & 7461 & 0 & 10705 & 13905 & 20250 \\
\hline
\end{tabular}

Table 5 Configuration cost optimization [CC part B]—raw result costs (optimality gap 0.05)

\begin{tabular}{rrrrrlllllllrrrr}
\hline $\mathrm{t}$ & $\mathrm{oI}$ & $\mathrm{oE}$ & \multicolumn{1}{l}{$\mathrm{oc}$} & $\mathrm{ocI}$ & $\mathrm{ocE}$ & $\mathrm{cS}$ & \multicolumn{1}{c}{$\mathrm{dS}$} & $\mathrm{aS}$ & $\mathrm{df}$ & $\mathrm{af}$ & $\mathrm{op}$ & $\mathrm{ccp}$ & $\mathrm{dcp}$ & $\mathrm{acp}$ \\
\hline 1 & 2000 & 0 & 16500 & 200 & 0 & 300 & 1000 & 40600 & 913 & 21029 & 137 & 0 & 622 & 8142 \\
2 & 0 & 0 & 33000 & 200 & 0 & 300 & 1000 & 39900 & 1712 & 23096 & 275 & 0 & 545 & 16546 \\
3 & 0 & 0 & 49500 & 200 & 0 & 300 & 1000 & 46200 & 2510 & 31633 & 412 & 0 & 1076 & 26382 \\
4 & 0 & 0 & 49500 & 0 & 0 & 300 & 1000 & 53900 & 3309 & 36701 & 412 & 0 & 978 & 35791 \\
5 & 0 & 0 & 66000 & 200 & 0 & 300 & 1000 & 58800 & 3652 & 46279 & 549 & 0 & 981 & 42561 \\
6 & 0 & 0 & 66000 & 0 & 0 & 300 & 1000 & 62300 & 4338 & 55634 & 549 & 0 & 1342 & 48543 \\
7 & 2000 & 0 & 82500 & 200 & 0 & 300 & 1000 & 63000 & 4908 & 61929 & 686 & 0 & 1186 & 57240 \\
8 & 0 & 0 & 99000 & 200 & 0 & 300 & 1000 & 67200 & 5709 & 69092 & 824 & 0 & 1279 & 67161 \\
9 & 0 & 0 & 115500 & 200 & 0 & 300 & 1000 & 72800 & 6854 & 79213 & 961 & 0 & 1629 & 76814 \\
10 & 0 & 0 & 132000 & 200 & 0 & 300 & 1000 & 74900 & 7197 & 91851 & 1098 & 0 & 1772 & 83400 \\
11 & 2000 & 0 & 148500 & 200 & 0 & 300 & 1000 & 65100 & 7768 & 102271 & 1235 & 0 & 1905 & 91073 \\
12 & 0 & 0 & 148500 & 0 & 0 & 300 & 1000 & 73500 & 8683 & 108059 & 1235 & 0 & 1980 & 99298 \\
13 & 0 & 0 & 165000 & 200 & 0 & 300 & 1000 & 67200 & 9368 & 114749 & 1373 & 0 & 2232 & 106976 \\
14 & 0 & 0 & 181500 & 200 & 0 & 300 & 1000 & 53900 & 10169 & 117551 & 1510 & 0 & 2318 & 115389 \\
15 & 0 & 0 & 198000 & 200 & 0 & 300 & 1000 & 46900 & 11085 & 121702 & 1647 & 0 & 2465 & 123204 \\
16 & 0 & 0 & 198000 & 0 & 0 & 300 & 1000 & 31500 & 11429 & 121930 & 1647 & 0 & 2374 & 129000 \\
\hline
\end{tabular}

and lacks the transition related cost component; however the scale of reconfigurations between consecutive periods is limited in this case by the assumption (enforced by additional constraints) that only pure incremental reconfigurations are admissible (see Sect. 1), i.e., that equipment, once installed, cannot be extracted from the network.

Raw results of each of the three experiments are presented in a dedicated table pair-(Tables 4, 5), (Tables 6, 7) and (Tables 8, 9) for, respectively CC, CTC, and CCPIR experiments. The first table of a pair (denoted as part
A) contains a subset of columns which is complemented by columns of the second table (part $B$ ). All these tables have 16 rows - each row is associated with a single timeperiod indicated in the first column, denoted by $t$. Columns $h$, hi, and hvi represent: total demand (required number of optical signals), total installed service capacity (number of tributary ports of connected access splitters), and total installed virtual service capacity (number of tributary ports of installed virtual access splitters-see Sect. 5), respectively. Next three column quadruples, i.e., $(c s, c s v, c s I, c s E),(d s$, 
Table 6 Configuration and transition cost optimization [CTC part A]—raw result costs (optimality gap 0.05)

\begin{tabular}{|c|c|c|c|c|c|c|c|c|c|c|c|c|c|c|c|c|}
\hline $\mathrm{t}$ & $\mathrm{h}$ & hi & hvi & $\mathrm{cs}$ & $\operatorname{csv}$ & csI & $\mathrm{csE}$ & $\mathrm{ds}$ & dsv & dsI & $\mathrm{dsE}$ & as & asv & asI & asE & o \\
\hline 1 & 247 & 508 & 21 & 8 & 4 & 420 & 0 & 173 & 0 & 1760 & 0 & 751 & 41 & 7585 & 0 & 6750 \\
\hline 2 & 571 & 1019 & 32 & 16 & 5 & 315 & 0 & 190 & 0 & 340 & 0 & 1298 & 46 & 6855 & 2100 & 6750 \\
\hline 3 & 952 & 1528 & 19 & 24 & 4 & 245 & 0 & 230 & 0 & 460 & 0 & 1837 & 34 & 6965 & 2280 & 6750 \\
\hline 4 & 1279 & 1782 & 12 & 28 & 5 & 175 & 0 & 345 & 0 & 1115 & 0 & 2185 & 25 & 3740 & 585 & 6750 \\
\hline 5 & 1542 & 2045 & 10 & 32 & 6 & 175 & 0 & 376 & 12 & 415 & 0 & 2561 & 14 & 4090 & 585 & 6750 \\
\hline 6 & 1827 & 2476 & 21 & 39 & 5 & 210 & 0 & 403 & 0 & 270 & 0 & 3043 & 34 & 6030 & 1245 & 13500 \\
\hline 7 & 2220 & 3034 & 29 & 48 & 5 & 315 & 0 & 459 & 20 & 775 & 0 & 3707 & 43 & 7825 & 1805 & 13500 \\
\hline 8 & 2666 & 3378 & 81 & 53 & 6 & 210 & 0 & 486 & 20 & 315 & 0 & 4161 & 107 & 6330 & 2055 & 13500 \\
\hline 9 & 3102 & 3896 & 69 & 61 & 5 & 245 & 0 & 562 & 0 & 655 & 85 & 4814 & 98 & 7445 & 2005 & 13500 \\
\hline 10 & 3464 & 4090 & 57 & 64 & 6 & 140 & 0 & 575 & 10 & 205 & 0 & 5115 & 77 & 3390 & 1070 & 13500 \\
\hline 11 & 3788 & 4520 & 30 & 71 & 5 & 210 & 0 & 631 & 0 & 485 & 0 & 5630 & 54 & 6920 & 3280 & 20250 \\
\hline 12 & 4113 & 4812 & 58 & 76 & 4 & 140 & 0 & 689 & 0 & 600 & 0 & 6003 & 96 & 5405 & 2075 & 20250 \\
\hline 13 & 4466 & 5108 & 86 & 80 & 5 & 175 & 0 & 718 & 4 & 355 & 0 & 6358 & 137 & 5320 & 1790 & 20250 \\
\hline 14 & 4855 & 5607 & 47 & 88 & 6 & 315 & 0 & 763 & 16 & 575 & 0 & 6934 & 76 & 6970 & 2970 & 20250 \\
\hline 15 & 5249 & 5985 & 71 & 94 & 6 & 210 & 0 & 739 & 12 & 445 & 1325 & 7332 & 114 & 5700 & 2170 & 20250 \\
\hline 16 & 5531 & 6102 & 8 & 96 & 5 & 35 & 0 & 746 & 4 & 0 & 0 & 7508 & 12 & 830 & 205 & 20250 \\
\hline
\end{tabular}

Table 7 Configuration and transition cost optimization [CTC part B]—raw result costs (optimality gap 0.05)

\begin{tabular}{|c|c|c|c|c|c|c|c|c|c|c|c|c|c|c|}
\hline $\mathrm{t}$ & oI & $\mathrm{oE}$ & oc & ocI & ocE & $\mathrm{cS}$ & $\mathrm{dS}$ & $\mathrm{aS}$ & $\mathrm{df}$ & af & op & ccp & $\mathrm{dcp}$ & acp \\
\hline 1 & 2000 & 0 & 16500 & 200 & 0 & 300 & 1000 & 40600 & 2019 & 19134 & 137 & 0 & 652 & 11648 \\
\hline 2 & 0 & 0 & 33000 & 200 & 0 & 300 & 500 & 16100 & 3459 & 23062 & 275 & 0 & 728 & 23233 \\
\hline 3 & 0 & 0 & 49500 & 200 & 0 & 300 & 1000 & 23100 & 4794 & 28591 & 412 & 0 & 845 & 33983 \\
\hline 4 & 0 & 0 & 66000 & 200 & 0 & 300 & 1000 & 21700 & 5659 & 37250 & 549 & 0 & 1245 & 40568 \\
\hline 5 & 0 & 0 & 66000 & 0 & 0 & 300 & 1000 & 25900 & 6343 & 46602 & 549 & 0 & 1334 & 45499 \\
\hline 6 & 2000 & 0 & 82500 & 200 & 0 & 300 & 1000 & 23100 & 7427 & 55459 & 686 & 0 & 1448 & 54511 \\
\hline 7 & 0 & 0 & 99000 & 200 & 0 & 300 & 1000 & 30100 & 8762 & 64421 & 824 & 0 & 1621 & 65164 \\
\hline 8 & 0 & 0 & 115500 & 200 & 0 & 300 & 1000 & 30100 & 9591 & 72511 & 961 & 0 & 1713 & 72486 \\
\hline 9 & 0 & 0 & 132000 & 200 & 0 & 300 & 1000 & 30100 & 11035 & 81195 & 1098 & 0 & 1991 & 83462 \\
\hline 10 & 0 & 0 & 132000 & 0 & 0 & 300 & 1000 & 22400 & 11360 & 91236 & 1098 & 0 & 2026 & 87883 \\
\hline 11 & 2000 & 0 & 148500 & 200 & 0 & 300 & 1000 & 32900 & 12694 & 100095 & 1235 & 0 & 2181 & 97708 \\
\hline 12 & 0 & 0 & 165000 & 200 & 0 & 300 & 1000 & 26600 & 13379 & 108273 & 1373 & 0 & 2390 & 104175 \\
\hline 13 & 0 & 0 & 165000 & 0 & 0 & 300 & 1000 & 25200 & 13884 & 114619 & 1373 & 0 & 2484 & 110343 \\
\hline 14 & 0 & 0 & 181500 & 200 & 0 & 300 & 1000 & 23800 & 15292 & 119479 & 1510 & 0 & 2614 & 120996 \\
\hline 15 & 0 & 0 & 198000 & 200 & 0 & 300 & 1000 & 18200 & 16411 & 121665 & 1647 & 0 & 2502 & 127192 \\
\hline 16 & 0 & 0 & 198000 & 0 & 0 & 300 & 0 & 3500 & 16446 & 125616 & 1647 & 0 & 2523 & 130878 \\
\hline
\end{tabular}

$d s v, d I, d E$ ), and (as, asv, as I, as E), expose: (i) total cost of installed splitters, (ii) installed virtual splitters, (iii) installation of new splitters, (iv) extraction of installed splitter in, respectively, central (c), distribution (d), and access (a) sites. Next two column triples- $(o, o I, o E)$ and $(o c, o c I$, $o c E$ )—show: (i) cost of installed equipment, (ii) installation cost, (iii) extraction cost associated with, respectively, OLT devices and OLT cards. The three columns $-c S, d S$, and $a S$-denote expenditures related to site survey to be executed in, respectively, central, distribution, and access site.
Columns $d f$ and af show charges to be paid for exploitation of trunk and distribution fibre paths, then column $o p$ indicates charges for allocation of OLT ports. Finally, column triple (ocp, $d c p, a c p)$ shows costs associated with occupied cabinet space in central, distribution, and access sites.

Information presented in these tables, despite being detailed and illustrative, does not directly show general trends. To facilitate this, we prepared more synthetic Tables 10,11 , and 12 that present accumulated costs grouped into 
Table 8 Configuration cost optimization, pure incremental reconfigurations [CC-PIR part A]—raw result costs (optimality gap 0.05)

\begin{tabular}{rrrrrrrrrrrrrrrrrrr}
\hline $\mathrm{t}$ & \multicolumn{1}{l}{$\mathrm{h}$} & $\mathrm{hi}$ & \multicolumn{1}{c}{$\mathrm{hvi}$} & $\mathrm{cs}$ & $\mathrm{csv}$ & \multicolumn{1}{c}{$\mathrm{csI}$} & $\mathrm{csE}$ & $\mathrm{ds}$ & $\mathrm{dsv}$ & $\mathrm{dsI}$ & $\mathrm{dsE}$ & $\mathrm{as}$ & asv & asI & asE & o \\
\hline 1 & 247 & 1024 & 0 & 16 & 6 & 770 & 0 & 200 & 10 & 2270 & 0 & 1229 & 0 & 12375 & 0 & 6750 \\
2 & 571 & 1249 & 24 & 20 & 4 & 70 & 0 & 206 & 0 & 125 & 125 & 1497 & 39 & 3040 & 0 & 6750 \\
3 & 952 & 1534 & 24 & 24 & 6 & 210 & 0 & 250 & 4 & 450 & 0 & 1876 & 43 & 3715 & 0 & 6750 \\
4 & 1279 & 2010 & 33 & 32 & 6 & 280 & 0 & 312 & 0 & 620 & 0 & 2470 & 61 & 6010 & 0 & 6750 \\
5 & 1542 & 2265 & 15 & 36 & 4 & 70 & 0 & 330 & 4 & 190 & 0 & 2851 & 26 & 3510 & 0 & 13500 \\
6 & 1827 & 2559 & 57 & 40 & 7 & 245 & 0 & 496 & 10 & 1705 & 0 & 3191 & 91 & 4285 & 0 & 13500 \\
7 & 2220 & 3053 & 69 & 48 & 4 & 175 & 0 & 404 & 69 & 390 & 1325 & 3796 & 116 & 6335 & 0 & 13500 \\
8 & 2666 & 3431 & 151 & 54 & 5 & 245 & 0 & 478 & 10 & 920 & 1325 & 4283 & 221 & 5910 & 0 & 13500 \\
9 & 3102 & 3841 & 39 & 61 & 6 & 280 & 0 & 554 & 73 & 1360 & 0 & 4862 & 64 & 4185 & 0 & 13500 \\
10 & 3464 & 4083 & 58 & 64 & 4 & 35 & 0 & 609 & 0 & 130 & 685 & 5191 & 96 & 3665 & 0 & 13500 \\
11 & 3788 & 4501 & 71 & 71 & 4 & 245 & 0 & 693 & 10 & 885 & 0 & 5750 & 134 & 5975 & 0 & 20250 \\
12 & 4113 & 4758 & 48 & 76 & 3 & 140 & 0 & 749 & 10 & 540 & 0 & 6160 & 95 & 3580 & 0 & 20250 \\
13 & 4466 & 5053 & 80 & 80 & 2 & 105 & 0 & 781 & 0 & 205 & 0 & 6571 & 147 & 4605 & 0 & 20250 \\
14 & 4855 & 5324 & 78 & 84 & 6 & 280 & 0 & 909 & 69 & 1920 & 0 & 6926 & 140 & 3620 & 0 & 20250 \\
15 & 5249 & 5632 & 95 & 88 & 3 & 35 & 0 & 923 & 0 & 130 & 1325 & 7358 & 159 & 4595 & 0 & 20250 \\
16 & 5531 & 5972 & 115 & 94 & 4 & 245 & 0 & 1002 & 0 & 685 & 0 & 7822 & 183 & 4960 & 0 & 20250 \\
\hline
\end{tabular}

Table 9 Configuration cost optimization, pure incremental reconfigurations [CC-PIR part B]—raw result costs (optimality gap 0.05)

\begin{tabular}{rrrrrrrrrrrrrrrr}
\hline $\mathrm{t}$ & $\mathrm{oI}$ & $\mathrm{oE}$ & \multicolumn{1}{c}{$\mathrm{oc}$} & $\mathrm{ocI}$ & $\mathrm{ocE}$ & \multicolumn{1}{c}{$\mathrm{cS}$} & \multicolumn{1}{c}{$\mathrm{dS}$} & $\mathrm{aS}$ & $\mathrm{df}$ & $\mathrm{af}$ & $\mathrm{op}$ & $\mathrm{ccp}$ & $\mathrm{dcp}$ & $\mathrm{acp}$ \\
\hline 1 & 2000 & 0 & 33000 & 400 & 0 & 300 & 1000 & 40600 & 3504 & 20070 & 275 & 0 & 751 & 24791 \\
2 & 0 & 0 & 49500 & 200 & 0 & 300 & 500 & 11200 & 4032 & 23636 & 412 & 0 & 779 & 28948 \\
3 & 0 & 0 & 49500 & 0 & 0 & 300 & 1000 & 17500 & 4848 & 30214 & 412 & 0 & 894 & 34385 \\
4 & 0 & 0 & 66000 & 200 & 0 & 300 & 1000 & 23800 & 6303 & 39189 & 549 & 0 & 1103 & 45149 \\
5 & 2000 & 0 & 82500 & 200 & 0 & 300 & 500 & 23800 & 6479 & 49751 & 686 & 0 & 1146 & 51538 \\
6 & 0 & 0 & 82500 & 0 & 0 & 300 & 1000 & 18900 & 7615 & 57054 & 686 & 0 & 1819 & 57814 \\
7 & 0 & 0 & 99000 & 200 & 0 & 300 & 1000 & 25900 & 8399 & 67562 & 824 & 0 & 1379 & 67194 \\
8 & 0 & 0 & 115500 & 200 & 0 & 300 & 1000 & 26600 & 9567 & 76061 & 961 & 0 & 1672 & 75174 \\
9 & 0 & 0 & 132000 & 200 & 0 & 300 & 1000 & 24500 & 10703 & 87592 & 1098 & 0 & 1902 & 83203 \\
10 & 0 & 0 & 132000 & 0 & 0 & 300 & 1000 & 21700 & 11167 & 98671 & 1098 & 0 & 2080 & 88587 \\
11 & 2000 & 0 & 148500 & 200 & 0 & 300 & 1000 & 34300 & 12015 & 111128 & 1235 & 0 & 2357 & 98373 \\
12 & 0 & 0 & 165000 & 200 & 0 & 300 & 1000 & 24500 & 12831 & 122355 & 1373 & 0 & 2553 & 105304 \\
13 & 0 & 0 & 165000 & 0 & 0 & 300 & 1000 & 26600 & 13471 & 129435 & 1373 & 0 & 2635 & 111922 \\
14 & 0 & 0 & 181500 & 200 & 0 & 300 & 1000 & 18900 & 14575 & 135625 & 1510 & 0 & 3145 & 118456 \\
15 & 0 & 0 & 181500 & 0 & 0 & 300 & 500 & 26600 & 14863 & 147356 & 1510 & 0 & 3185 & 125673 \\
16 & 0 & 0 & 198000 & 200 & 0 & 300 & 1000 & 26600 & 15967 & 155592 & 1647 & 0 & 3425 & 132436 \\
\hline
\end{tabular}

four categories. The first category, denoted as $I-N$, illustrates total charge for infrastructure resources (trunk and distribution fibres; central, distribution. and access cabinet space; central OLT port allocation) the NP role has to pay at the $I-N$ interface. Second category of costs, denoted as $E-N$, describes accumulated charge the NP role pays at the $E-$ $N$ interface for leasing passive (optical splitters) and active (OLT devices and OLT cards) equipment. The third category, denoted by $H$, indicates accumulated costs of configuration transitions (installation/extraction of equipment and site sur- veys). Finally, the fourth category shows the accumulated total expenditures.

As we mention in the beginning of this section, we consider results of the CTC experiment as a reference solution for the remaining two. Following this way, we prepared Figs. 13 and 14 that illustrate relations between results of, respectively, the $\mathrm{CC}$ experiment and the CC-PIR experiments to the reference solution. Each figure shows four curves that refer to particular cost categories identified above; within 
Table 10 CC experiment-accumulated costs by category

\begin{tabular}{lrrrr}
\hline Period & $I-N$ cost & $E-N$ cost & \multicolumn{1}{l}{$H$ cost } & Total \\
\hline 1 & 30844 & 23445 & 51470 & 105759 \\
2 & 73017 & 63841 & 107875 & 244733 \\
3 & 135030 & 121349 & 175555 & 431934 \\
4 & 212220 & 179289 & 253695 & 645203 \\
5 & 306242 & 254161 & 329335 & 889737 \\
6 & 416648 & 329516 & 413710 & 1159874 \\
7 & 542598 & 428478 & 502425 & 1473501 \\
8 & 686662 & 544533 & 595670 & 1826865 \\
9 & 852133 & 677781 & 695905 & 2225819 \\
10 & 1037451 & 828215 & 799415 & 2665081 \\
11 & 1241703 & 1002355 & 887500 & 3131558 \\
12 & 1460958 & 1177046 & 988425 & 3626429 \\
13 & 1695656 & 1368839 & 1081390 & 4145885 \\
14 & 1942593 & 1577622 & 1156925 & 4677140 \\
15 & 2202696 & 1803485 & 1226465 & 5232646 \\
16 & 2469076 & 2029820 & 1285005 & 5783901 \\
\hline
\end{tabular}

Table 11 CTC experiment-accumulated costs by category

\begin{tabular}{lrrrr}
\hline Period & $I-N$ cost & $E-N$ cost & $H$ cost & \multicolumn{1}{l}{ Total } \\
\hline 1 & 33591 & 23476 & 53865 & 110932 \\
2 & 84347 & 64233 & 80575 & 229155 \\
3 & 152972 & 122073 & 115125 & 390169 \\
4 & 238243 & 197062 & 143940 & 579245 \\
5 & 338571 & 272437 & 176405 & 787413 \\
6 & 458102 & 371480 & 210760 & 1040342 \\
7 & 598894 & 487597 & 253080 & 1339571 \\
8 & 756156 & 620977 & 293590 & 1670723 \\
9 & 934938 & 771364 & 335625 & 2041927 \\
10 & 1128542 & 922409 & 364130 & 2415081 \\
11 & 1342455 & 1097036 & 411425 & 2850916 \\
12 & 1572045 & 1288780 & 447745 & 3308570 \\
13 & 1814747 & 1480977 & 481885 & 3777609 \\
14 & 2074638 & 1690034 & 518015 & 4282687 \\
15 & 2344055 & 1916182 & 547565 & 4807802 \\
16 & 2621165 & 2142627 & 552435 & 5316227 \\
\hline
\end{tabular}

each category, series of data points are referring to points returned by the CTC experiment.

Results illustrated in Figs. 13 and 14 lead to the following conclusions. First, results of the CC experiment (see Fig. 13) perfectly adhere to our expectations - the experiment gives the best infrastructure cost (c.a. $90 \%$ of infrastructure cost provided by CTC) and very costly reconfiguration (c.a. $230 \%$ w.r.t. the same cost of the reference) what leads to the total cost about $10 \%$ greater than in the reference case. Second, analysis of the CC-PIR experiment (in Fig. 14) indicates that
Table 12 CC-PIR experiment—accumulated costs by category

\begin{tabular}{lrrrr}
\hline Period & $I-N$ cost & $E-N$ cost & $H$ cost & \multicolumn{1}{l}{ Total } \\
\hline 1 & 49390 & 39981 & 59715 & 149086 \\
2 & 107197 & 97730 & 75275 & 280201 \\
3 & 177949 & 155804 & 98450 & 432203 \\
4 & 270243 & 230841 & 130660 & 631744 \\
5 & 379844 & 329711 & 161230 & 870785 \\
6 & 504833 & 429206 & 187665 & 1121704 \\
7 & 650190 & 545538 & 223290 & 1419019 \\
8 & 813625 & 679101 & 259790 & 1752517 \\
9 & 998123 & 829642 & 291615 & 2119380 \\
10 & 1199727 & 980776 & 319130 & 2499633 \\
11 & 1424835 & 1155629 & 364035 & 2944499 \\
12 & 1669251 & 1347561 & 394295 & 3411107 \\
13 & 1928086 & 1539981 & 427110 & 3895177 \\
14 & 2201397 & 1749511 & 453330 & 4404238 \\
15 & 2493984 & 1959360 & 486815 & 4940160 \\
16 & 2803052 & 2186251 & 520805 & 5510108 \\
\hline
\end{tabular}

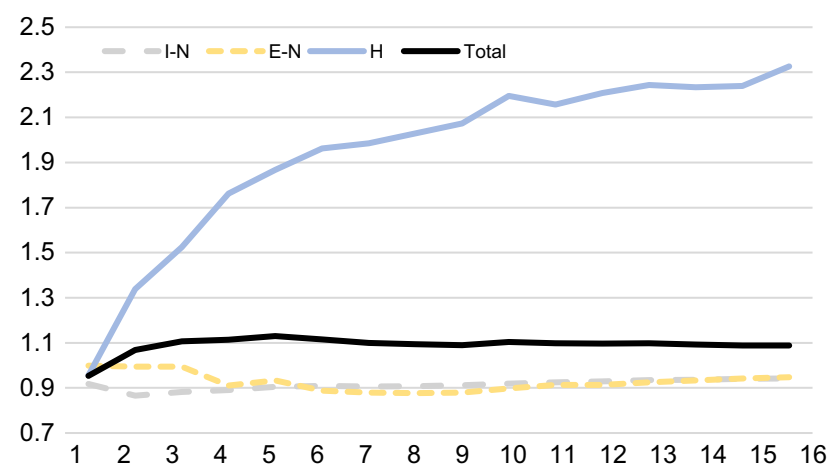

Fig. 13 CC experiment-quality of results w.r.t. CTC

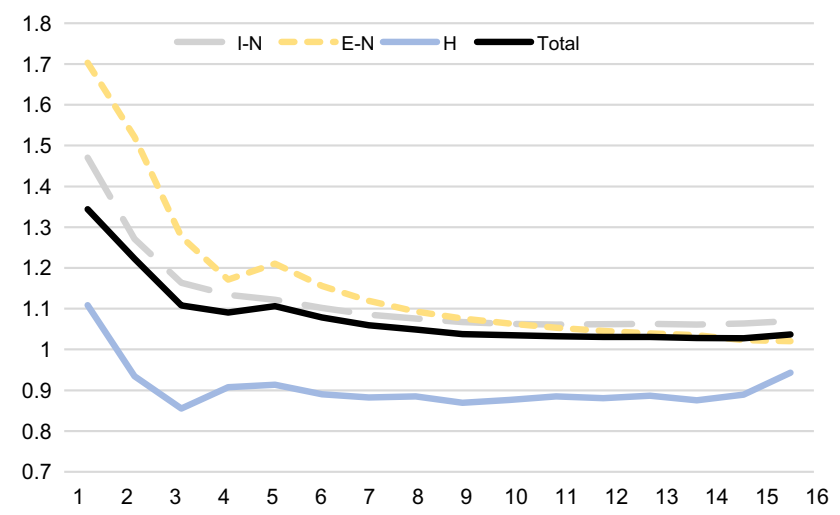

Fig. 14 CC-PIR experiment-quality of results w.r.t. CTC

the approach (no transition cost in the objective function, solely pure incremental reconfiguration) can lead to surprisingly good results. The real transition costs are generally at the level $10 \%$ below this of the reference solution, infrastruc- 
ture costs ( $I-N$ and $E-N$ categories) are a bit higher, still the total cost, in the long term run, approaches the optimal (reference) one. This suggest that the CC-PIR approach could be considered as a simpler, but still very attractive, alternative for the reference approach.

\section{Conclusions}

In this paper, we present results of our research on models and methods for designation of optimal trajectory for the multi-period evolution of FTTx networks. We propose business and cost models suitable for analysis of multi-period network evolution. We formulate the MIP optimization problem to designate the most cost-effective evolution pattern for a given sequence of demand vectors. The presented approach has been implemented and tested. Results of selected numerical experiments that prove validity of the approach has been given.

In our opinion, the approach presented in this paper is interesting and worth further investigations. In the next steps, we plan to increase computational efficiency of our implementation to shorten computation times, decrease achievable optimality gaps, and increase the number of considered time periods. Having this achieved, we will have the possibility to analyze larger and more complex cases than these presented in our numerical experiments. As we mention in Sect. 5, the problem has a special structure, which seems to be a promising starting point for further activities.

Acknowledgements This research was supported by National Science Centre, Poland, under Grant 2014/15/D/ST7/05320 "Open problems in large-scale optical access network design".

\section{Compliance with ethical standards}

Conflict of interest All authors declare that they have no conflict of interest.

Open Access This article is distributed under the terms of the Creative Commons Attribution 4.0 International License (http://creativecomm ons.org/licenses/by/4.0/), which permits unrestricted use, distribution, and reproduction in any medium, provided you give appropriate credit to the original author(s) and the source, provide a link to the Creative Commons license, and indicate if changes were made.

\section{References}

1. Angilella, V., Chardy, M., \& Ben-Ameur, W. (2016). Cables network design optimization for the fiber to the home. In 12th international conference on the design of reliable communication networks (DRCN) (pp. 87-94).

2. Arévalo, G. V., \& Gaudino, R. (2017). A techno-economic network planning tool for PON deployment including protection strategies.
In 19th international conference on transparent optical networks (ICTON), 2017 (pp. 1-4).

3. Arévalo, G. V., Hincapié, R. C., \& Gaudino, R. (2017). Optimization of multiple PON deployment costs and comparison between GPON, XGPON, NGPON2 and UDWDM PON. Optical Switching and Networking, 25(Supplement C), 80-90.

4. Bihimani, A., Horngren, C. T., Datar, S. M., \& Foster, G. (2008). Management and cost accounting (4th ed.). London: Pearson Education Limited.

5. Bisiani, R. (1987). Encyclopedia of artificial intelligence, chapter beam search (pp. 56-58). Hoboken: Wiley.

6. Bley, A. (2003). A Lagrangean approach for integreted network design and routing in IP networks. In Proceedings INOC'2003, Evry-Paris (pp. 107-113).

7. Bogaert, J. V. (2016). FTTH business guide (5th ed.). Technical report, FTTH Council Europe. www.ftthcouncil.eu. Accessed 23 Aug 2018.

8. Bull, E. C., \& Rigby, P. (Eds.). (2018). FTTH handbook. FTTH Council Europe: Technical report.

9. Chardy, M., Costa, M.-C., Faye, A., \& Trampont, M. (2012). Optimizing splitter and fiber location in a multilevel optical FTTH network. European Journal of Operational Research, 222, 430440.

10. Cobo, L., Chamberland, S., \& Ntareme, A. (2012). Low cost fiberto-the-node access network design. In Proceedings of the 15th international telecommunications network strategy and planning symposium (NETWORKS), Rome, Italy (pp. 1-4).

11. Courcoubetis, C., \& Weber, R. (2003). Pricing communication networks: Economics, technology and modelling. Hoboken: Wiley.

12. D'Andreagiovanni, F., Mett, F., Nardin, A., \& Pulaj, J. (2017). Integrating LP-guided variable fixing with MIP heuristics in the robust design of hybrid wired-wireless FTTx access networks. Applied Soft Computing, 61(Supplement C), 1074-1087.

13. Forzati, M., Mattsson, C., \& Larsen, C. P. (2014). Open access networks and Swedish market in 2013. Retrieved August 23, 2018 from https://www.acreo.se/sites/default/files/pub/acreo.se/ EXPERTISE/broadband/forzati-mattsson_-_vitel_2013.pdf.

14. Forzati, M., Mattsson, C., Corbet, M., \& Cullen, D. (2014). European commission. Technical report: Guide to high-speed broadband investment.

15. Fourer, R., Gay, D. M., \& Keringhan, B. W. (2003). AMPL: A modeling language for mathematical programming (2nd ed.). Sao Paulo: Duxbury-Thomson Learning.

16. Grötschel, M., Raack, C., \& Werner, A. (2014). Towards optimizing the deployment of optical access networks. EURO Journal on Computational Optimization, 2(1), 17-53.

17. Hervet, C., Faye, A., Costa, M.-C., Chardy, M., \& Francfort, S. (2013). Solving the two-stage robust FTTH network design problem under demand uncertainty. In Proceedings of the international network optimization conference, Costa Adeje, Spain.

18. Hervet, C., \& Chardy, M. (2012). Passive optical network design under operations administration and maintenance considerations. Journal of Applied Operational Research, 222(3), 152-172.

19. ITU-T. (2005). Broadband optical access systems based on passive optical networks (PON). Recommendation G.983.1. Geneva: International Telecommunication Union.

20. ITU-T. (2005). Generic functional architecture of transport networks. Recommendation G.805. Geneva: International Telecommunication Union.

21. ITU-T. (2008). Gigabit-capable passive optical networks (GPON): General characteristics. Recommendation G.984.1. Geneva: International Telecommunication Union.

22. Kokangul, A., \& Ari, A. (2011). Optimization of passive optical network planning. Applied Mathematical Modelling, 35(7), 33453354. 
23. Mycek, M., Pióro, M., \& Żotkiewicz, M. (2018). MIP model for efficient dimensioning of real-world FTTH trees. Telecommunication Systems, 68(2), 239-258.

24. Paltridge, S. (2015). Development of high speed networks and the role of municipal networks. Technical report. Organisation for Economic Co-operation and Development, report DSTI/ICCP/CISP(2015)1/FINAL.

25. Pióro, M., \& Medhi, D. (2004). Routing, flow, and capacity design in communication and computer networks. Burlington: MorganKaufmann Publishers.

26. Poon, K. F., \& Ouali, A. (2011). A MILP based design tool for FTTH access networks with consideration of demand growth. In Proceedings of the 6th international conference on internet technology and secured transactions, Abu Dhabi, United Arab Emirates.

27. Rogers, E. (2003). Diffusion of innovations. New York City: Simon and Schuster.

28. Sánchez, R., Hernández, J. A., García, J. M., \& Larrabeiti, D. (2016). Provisioning $1 \mathrm{~Gb} / \mathrm{s}$ symmetrical services with nextgeneration passive optical network technologies. IEEE Соттиnications Magazine, 54(2), 72-77.

29. Schneir, J. R., \& Xiong, Y. (2014). Cost analysis of network sharing in FTTH/PONs. IEEE Communications Magazine, 52(8), 126134.
30. Tahon, M., Ooteghem, J. V., Casier, K., Verbrugge, S., Colle, D., Pickavet, M., et al. (2014). Improving the FTTH business casseA joint telco-utility network rollout model. Telecommunications Policy, 38, 426-437.

31. van Loggerenberg, S. P., Grobler, M. J., \& Terblanche, S. E. (2012). Optimization of PON planning for FTTH deployment based on coverage. In Proceedings of the Southern Africa telecommunication networks and applications conference, Fancourt, South Africa.

32. Żotkiewicz, M., \& Mycek, M. (2016). Impact of demand uncertainty models on FTTH network design. In Proceedings of international conference on transparent optical networks ICTON 2016, Trento, Italy.

33. Żotkiewicz, M. (2018). Uncertainty in FTTH splitter installation. Networks, 71(2), 153-165.

34. Żotkiewicz, M., \& Mycek, M. (2017). Reducing the costs of FTTH networks by optimized splitter and OLT card deployment. IEEE/OSA Journal of Optical Communications and Networking, 9(5), 412-422.

35. Żotkiewicz, M., Mycek, M., \& Tomaszewski, A. (2016). Profitable areas in large-scale FTTH network optimization. Telecommunication Systems, 61(3), 591-608. 